Face à l'irresponsabilité : la dynamique de la solidarité

\title{
La responsabilité solidaire des États et/ou des organisations internationales : une institution négligée
}

\section{Samantha Besson}

DOI : 10.4000/books.cdf.5201

Éditeur : Collège de France

Lieu d'édition : Paris

Année d'édition : 2018

Date de mise en ligne : 19 mars 2018

Collection : Conférences

ISBN électronique : 9782722604803

\section{Q books}

http://books.openedition.org

\section{Édition imprimée}

Date de publication : 20 mars 2018

Ce document vous est offert par Collège de France

COLL E G E

DE FRANCE

Référence électronique

BESSON, Samantha. La responsabilité solidaire des États et/ou des organisations internationales : une institution négligée In : Face à l'irresponsabilité : la dynamique de la solidarité [en ligne]. Paris : Collège de France, 2018 (généré le 04 avril 2018). Disponible sur Internet : <http://books.openedition.org/ cdf/5201>. ISBN : 9782722604803 . DOI : 10.4000/books.cdf.5201. 


\title{
La responsabilité solidaire des États et/ou des organisations internationales: une institution négligée
}

\author{
Samantha Besson
}

Professeur de droit international public et de droit européen, université de Fribourg

\begin{abstract}
«Le fonctionnalisme a toujours souffert et souffre encore d'un biais important en faveur de l'organisation internationale. Il ne permet pas de rendre des comptes pour le rôle de l'organisation internationale dans ses relations à d'autres acteurs que les États membres de cette organisation, précisément parce que la fonction de l'organisation peut toujours être invoquée pour justifier toute activité de l'organisation et ce, même au détriment de tierces parties. C'est ce biais qui exige que le fonctionnalisme soit adapté en tant que théorie des organisations internationales, mais qui, en même temps, rend cette adaptation très difficile. » J. Klabbers, «The transformation of international organizations law», European Journal of International Law, vol. 26, $\mathrm{n}^{\circ}$ 1, 2015, p. 10 (traduction de l'auteur).
\end{abstract}

$\mathrm{L}$ A RESPONSABILITÉ INTERNATIONALE, ou de droit international, désigne l'ensemble des obligations (secondaires) de cessation, de non-répétition et de réparation des sujets de droit international que sont les États et les organisations internationales (OI), pour les violations d'obligations (primaires) de droit international qui peuvent leur être attribuées (art. 2 des Articles sur la responsabilité de l'État pour fait internationalement illicite [ARSIWA ] ${ }^{1}$; art. $3 \mathrm{du}$ Projet d'articles sur la responsabilité des organisations internationales [DARIO] ${ }^{2}$ ).

Le point de départ de la présente contribution est le concours ou la pluralité de responsabilités. Du fait des rapports sans cesse plus étroits entre États et/ou OI, en effet, les violations du droit international sont de plus en plus souvent le fait de plusieurs États et/ou OI qui contribuent ensemble à violer les droits des mêmes sujets et là causer un même préjudice.

1. Assemblée générale des Nations unies, «Résolution 56/83. Responsabilité de l’État pour fait internationalement illicite", 12 décembre 2001, A/RES/56/83 (en anglais: Articles on the Responsibility of States for Internationally Wrongful Acts, ARSIWA).

2. CDI, Projet d'articles sur la responsabilité des organisations internationales, 9 novembre 2011, A/66/10, $\$ 87$ (en anglais: Draft Articles on the Responsibility of International Organizations, DARIO). 
Se posent alors différentes questions épineuses quant à ce qui constitue une juste répartition des responsabilités entre ces différents responsables. Malgré leur importance grandissante en pratique, ces questions demeurent encore insuffisamment explorées ${ }^{3}$, et ce, notamment, relativement au fait internationalement illicite d'une ou plusieurs OI.

Il y a au moins quatre constellations dans lesquelles ces violations du droit international des droits de l'homme, qu'on peut qualifier de «transnationales» parce qu'elles impliquent plusieurs États et/ou OI, interviennent, et ce, de manière croissante. Il s'agira ici de se concentrer avant tout sur la responsabilité pour violation par plusieurs États et/ou OI de leurs obligations relevant du droit international des droits de l'homme (par ex. de la Convention européenne des droits de l'homme $[\mathrm{CEDH}])^{4}$. Ces constellations, sont:

- premièrement, les interventions transnationales de certains États et/ou OI qui les amènent à exercer leur juridiction sur les mêmes individus titulaires de droits de l'homme, y compris en dehors de leur territoire. On pensera, par exemple, à la participation de troupes du Royaume-Uni et des Pays-Bas à des opérations de maintien ou d'imposition de la paix en Serbie, au Kosovo ou en Irak (sous légide ou non de l'Organisation des Nations unies [ONU] et/ou de l'Union européenne [UE]). Un autre exemple est celui de la participation de soldats du Népal, malades du choléra, à la mission de maintien de la paix de l'ONU en Haïti après le tremblement de terre;

\footnotetext{
3. Voir, cependant, J.E Noyes. et B.D. Smith, «State responsibility and the principle of joint and several liability», Yale Journal of International Law, vol. 13, n² 2, 1988, p. 225-267; S. Besson, «La pluralité d'États responsables: vers une solidarité internationale?», Revue suisse de droit international et de droit européen, $\mathrm{n}^{\circ}$ 1, 2007, p. 13-38; A. Orakhelashvili, «Division of Reparation between Responsible Entities», in J. Crawford, A. Pellet et S. Olleson (dir.), The Law of International Responsibility, Oxford, Oxford University Press, 2010, p. 647-666; R.P. Alford, «Apportioning Responsibility among Joint Tortfeasors for International Law Violations ", Pepperdine Law Review, vol. 38, n², 2011, p. 233-255; A. Nollkaemper et D. Jacobs, «Shared responsibility in international law: A conceptual framework», Michigan Journal of International Law, vol. 34, n 2, 2013, p. 359-438; P. d'Argent, «Reparation, cessation, assurances and guarantees of non-repetition », in A. Nollkaemper et I. Plakokefalos (dir.), Principles of Shared Responsibility in International Law. An Appraisal of the State of the Art, Cambridge, Cambridge University Press, 2014, p. 208-250; A. Nollkaemper, «Introduction», in A. Nollkaemper et I. Plakokefalos (dir.), Principles of Shared Responsibility in International Law, op. cit., p. 1-24.

4. Cf. par exemple A. Khalfan, «Division of responsibility amongst states», in M. Langford et al. (dir.), Global Justice, State Duties: The Extraterritorial Scope of Economic, Social and Cultural Rights in International Law, Cambridge, Cambridge University Press, 2012, p. 299-331; M. Den Heijer, «Shared Responsibility Before the European Court of Human Rights ", Netherlands International Law Review, vol. 60, n³ 2013, p.411-440; W. Vandenhole, «Obligations and responsibility in a plural and diverse duty-bearer human rights regime», in W. Vandenhole (dir.), Challenging Territoriality in Human Rights Law - Building Blocks for a Plural and Diverse Duty-Bearer Regime, Londres, Routledge, 2015, p. 115-135; S. Besson, «L'application extra-territoriale des droits de l'homme internationaux en pratique: juridictions concurrentes, obligations conjointes et responsabilités partagées », in P. d'Argent (dir.), Droit des frontières internationales, Actes des Journées franco-allemandes de droit international 2014, Paris, Pedone, 2016, p. 245-259; S. Besson, «Concurrent responsibilities under the European Convention on human rights: The concurrence of human rights jurisdictions, duties and responsibilities ", in J. Motoc et A. von Aaken (dir.), The ECHR and General International Law, Oxford, Oxford University Press, 2018.
} 
- deuxièmement, les mouvements transnationaux (physiques ou virtuels) des titulaires de droits de l'homme eux-mêmes qui les amènent sous la juridiction de plusieurs États et/ou OI. On pensera, par exemple, à la surveillance par les États-Unis des données électroniques personnelles du chef d'État allemand ou encore du renvoi de migrants, en vertu du système de Dublin en droit des migrations de l'UE, de la Belgique vers la Hongrie ou la Grèce;

- troisièmement, la coopération transnationale entre États et/ou OI qui rend leurs droit et activités, même nationaux ou internes, interdépendants. On pensera, par exemple, à la mise en œuvre d'un mandat d'arrêt européen des autorités bulgares en Espagne ou encore à lapplication des standards de restructuration de dettes du Fonds monétaire international (FMI) en Grèce;

- quatrièmement, la nature transnationale de certains phénomènes, naturels ou non, qui impliquent nécessairement l’action et/ou lomission de plusieurs États et/ou OI. On mentionnera, par exemple, la gestion de catastrophes environnementales, des épidémies ou encore des marchés financiers par les États et/ou certaines OI, comme l'ONU ou le FMI.

En principe, la responsabilité internationale est (i) indépendante ou distincte et (ii) divise (art. 42 et 47[1] ARSIWA; art. 43 et 48[1] DARIO). Lorsque plusieurs États et/ou OI ont causé le même préjudice, chaque État et/ou OI responsable peut être recherché(e) individuellement et séparément (et non pas conjointement avec d'autres) (i), mais ne doit répondre de la violation de ses obligations internationales qu'à hauteur de sa contribution au préjudice causé (et non pas de l'entier de ce dernier) (ii). Peu importe à cet égard que d'autres États ou OI aient contribué à causer ce même préjudice par le même fait illicite ou par plusieurs faits illicites distincts (de même nature ou différents), d'une part, et que le préjudice ait été causé par la violation des mêmes obligations internationales ou non, d'autre part.

En cas de pluralité d'États et/ou d'OI impliqués dans un même préjudice, le droit international général de la responsabilité reconnaît, certes, comme le droit national de la responsabilité civile ${ }^{5}$, le principe de la responsabilité solidaire (art. $47 \mathrm{ARSIWA}^{6}$; art. $48 \mathrm{DARIO}^{7}$ ). Cette institution permet au sujet

\footnotetext{
5. Pour une comparaison du principe de solidarité des débiteurs en droit civil suisse et en droit international, voir S. Besson, op. cit., 2007, p. 13-14.

6. Art. 47 ARSIWA: «Pluralité d'États responsables. 1. Lorsque plusieurs États sont responsables du même fait internationalement illicite, la responsabilité de chaque État peut être invoquée par rapport à ce fait. 2. Le paragraphe 1: a) ne permet à aucun État lésé de recevoir une indemnisation supérieure au dommage qu'il a subi; b) est sans préjudice de tout droit de recours à l'égard des autres États responsables.» 7. Art. 48 DARIO: «Responsabilité d'une organisation internationale et d'un ou plusieurs États ou une ou plusieurs organisations internationales. 1. Lorsqu'une organisation internationale et un ou plusieurs États ou une ou plusieurs autres organisations internationales sont responsables du même fait internationalement illicite, la responsabilité de chaque État ou organisation internationale peut être invoquée par rapport à ce fait. 2. Une responsabilité subsidiaire peut être invoquée dans la mesure où l'invocation de la responsabilité principale n'a pas abouti à une réparation. 3. Les paragraphes 1 et 2 : a) ne permettent à aucun État ou organisation internationale lésés de recevoir une indemnisation supérieure au dommage subi ; b) sont sans préjudice de tout droit de recours que l'État ou organisation internationale ayant donné la réparation peuvent avoir à l'égard des autres États ou organisations internationales responsables.»
} 
lésé (État, OI ou particulier) (i) de ne rechercher qu'un(e) seul(e) des États et/ou des OI responsables (la responsabilité demeure donc individuelle ou indépendante), mais (ii) d'obtenir de ce dernier / de cette dernière réparation de l'ensemble de son préjudice (la responsabilité devient indivise) ${ }^{8}$.

La responsabilité solidaire n'a cependant qu'une applicabilité limitée en droit international, du fait, d'une part, de ses conditions très strictes, notamment celle de l'existence d'un même fait illicite à l'origine du préjudice (art. 47[1] ARSIWA; art. 48[1] DARIO) et, d'autre part, des modalités de mise en ouvre rigoureuses concernant l'interdiction du cumul d'actions et l'existence de recours internes (art. 47(2) ARSIWA; art. 48(2) DARIO). En outre, alors même qu'il est applicable, le principe de responsabilité solidaire n'est que rarement mis en œuvre en pratique. Seuls certains régimes spéciaux de responsabilité en droit international l'ont adopté et en ont précisé les modalités. Et en droit international des droits de l'homme, la responsabilité solidaire ne trouve aucune application, même lorsque ses conditions d'application sont remplies.

Il s'agira dans cette contribution d'explorer les raisons de ce manque d'applicabilité, voire d'application de la responsabilité solidaire et d'identifier les moyens d'y remédier. Cette situation est en effet source d'injustice. C'est le cas tout d'abord pour les victimes individuelles des violations. Il existe un risque de réparation incomplète du préjudice subi en fonction de l'État / de l'OI recherché(e) en responsabilité. Chacun d'entre eux répondra pour sa part de responsabilité seulement, et pour autant qu'on puisse faire reconnaître cette responsabilité, bien sûr. La situation est aussi source d'injustice pour les auteurs des violations. Il existe un risque d'enrichissement illégitime de l'État / de l'OI qui n'est pas recherché(e) en responsabilité au détriment de celui/ceux qui l'est/le sont, et, à l'inverse, un risque pour l'État / l'OI recherché(e) d'encourir une responsabilité supérieure à sa responsabilité de fait, et ce parce qu'il/elle est le seul/la seule pour lequel/laquelle un effort d'attribution est réalisé, avec les risques de distribution injuste de responsabilité que cela implique.

8. À cet égard, il faut bien distinguer la notion de responsabilité solidaire développée sur cette base, dans cette contribution, de celle qu'utilise Alain Supiot, dans son introduction (voir aussi A. Supiot, «Introduction», in A. Supiot et M. Delmas-Marty (dir.), Prendre la responsabilité au sérieux, Paris, PUF, 2015, p.9-35; A. Supiot, «Introduction», in A. Supiot (dir.), La Solidarité. Enquête sur un principe juridique, Paris, Collège de France / Odile Jacob, 2015, p.7-32). Parmi les formes qu'elle peut prendre selon lui (par exemple la responsabilité causale ou pour le fait d'autrui), toutes ne cadrent pas avec la structure de la responsabilité de droit international ou d'une manière différente. Ainsi, comme nous le verrons, la première est étrangère à la nature objective, et donc nécessairement causale, de la responsabilité internationale (pour fait internationalement illicite), tandis que la seconde constitue une forme de responsabilité par attribution (de responsabilité) qui se trouve déjà au cœur de la responsabilité internationale, y compris solidaire. Par contre, la responsabilité solidaire des États membres d'une OI pour violation d'une obligation de diligence est l'une des propositions développées en section 4 . 
Le nombre croissant de concours potentiels de responsabilités en pratique, que ce soit du fait des situations transnationales mentionnées précédemment ou, plus simplement, de l'extension du champ d'activités des OI, rend la question de la répartition juste des responsabilités entre plusieurs États/OI d'autant plus pressante. Après tout, on sait encore très peu quels critères utiliser pour répartir les responsabilités en cas de causalité complexe ou en cas de responsabilités plurales par attribution de responsabilité, principalement lorsqu'est impliquée la responsabilité des OI, mais pas uniquement. C'est d'ailleurs une difficulté qui a été identifiée depuis longtemps déjà par les théoriciens de la justice globale. On pensera, par exemple, aux discussions qui portent sur les obligations, mais aussi sur les responsabilités de plusieurs États et OI en matière de justice climatique ou économique ${ }^{9}$. Il est donc intéressant de revenir à la question de la responsabilité solidaire en droit international et d'en identifier non plus seulement les limites, comme il a toujours été de bon ton de le faire ${ }^{10}$, mais aussi le potentiel.

En réponse à cette difficulté, ma proposition sera d'interpréter le droit international de la responsabilité de manière à étendre l'applicabilité de la responsabilité solidaire aux violations transnationales du droit international des droits de l'homme. Cela permettrait d'inclure non seulement les cas où le préjudice est causé par un même fait illicite conjoint, comme cela peut être déjà le cas maintenant (article 47[1] ARSIWA / article 48[1] DARIO), mais aussi lorsqu'il y a eu plusieurs faits illicites distincts, mais que la causalité est complexe et que cela rend la répartition des responsabilités individuelles difficile à établir dans le régime de la responsabilité divise.

Concrètement, après quelques éléments introductifs sur les catégories du droit de la responsabilité internationale (et notamment les formes d'attribution de comportement/responsabilité) et quelques clarifications terminologiques des notions encore vagues de responsabilités "plurales", «partagées» et «solidaires», la contribution s'articulera en trois points: une présentation du problème posé par le régime actuel de répartition des responsabilités en cas de responsabilité partagée des États et/ou des OI, qu'elle soit concurrente ou conjointe; une proposition relative à l'extension du champ d'application de la responsabilité solidaire à de nouvelles circonstances et à son régime de mise en œuvre; et une distinction entre cette proposition et une proposition additionnelle relative à la responsabilité subsidiaire et collective des États membres d'une OI relativement au fait illicite et à la responsabilité

\footnotetext{
9. Cf. par exemple D. Miller, «Distributing responsibilities», Journal of Political Philosophy, 2001, vol. 9, n 4, p. 453-471; H. Shue, «Transboundary Damage in Climate Change: Criteria for Allocating Responsibility", in A. Nollkaemper et D. Jacobs (dir.), Distribution of Responsibilities in International Law, Cambridge, Cambridge University Press, 2015, p. 321-340.

10. Cf. par exemple J. Crawford, State Responsibility: The General Part, Cambridge, Cambridge University Press, 2013, p.333 sq.
} 
principale de cette dernière. L'argument se terminera par une conclusion critique du régime général de la responsabilité internationale des États et des OI, et notamment du fonctionnalisme qui le caractérise.

\section{La responsabilité de droit international: sources, sujets, conditions et contenu}

Pour rappel, la responsabilité internationale désigne l'ensemble des obligations (secondaires ou "responsabilités» stricto sensu) de cessation, de non-répétition et de réparation dues par les sujets de droit international que sont les États et les OI pour les violations des obligations (primaires) de droit international qui peuvent leur être attribuées.

Il s'agit donc d'une responsabilité pour fait illicite (art. 1 ARSIWA; art. 3 DARIO), qu'il convient de distinguer de la responsabilité pour fait licite (accountability ou liability), cette dernière étant presque inexistante en droit international ${ }^{11}$. Il est intéressant de remarquer à cet égard que la structure de la responsabilité internationale pour fait illicite, et notamment son fondement dans la violation objective du droit et, par conséquent, l'exclusion de la faute de ses conditions, exclut d'emblée la possibilité d'une responsabilité causale, ou, du moins, d'une responsabilité causale qui puisse être valablement distinguée de la première sur la base de l'absence de faute ou de la négligence comme en droit interne ${ }^{12}$. Cela rend d'ailleurs, comme nous le verrons, d'autant plus incompréhensible le peu d'intérêt existant pour la causalité dans le droit actuel de la responsabilité internationale.

L'objet de cette section est de présenter, de manière sommaire, les sources, les sujets, les conditions et le contenu de la responsabilité internationale, et ce, afin de préparer la discussion du régime applicable en cas de pluralité d'États et/ou d'OI responsables. Ces précisions sont d'autant plus importantes que la structure de la responsabilité en droit international est différente ou, du moins, hybride par rapport à celle que l'on rencontre dans les traditions nationales.

\footnotetext{
11. Cf. par exemple A. Boyle, «Liability for injurious consequences of acts not prohibited by international law», in J. Crawford, A. Pellet et S. Olleson (dir.), The Law of International Responsibility, Oxford, Oxford University Press, 2010, p. 95-104.

12. Le fondement de la distinction entre responsabilité internationale pour fait internationalement illicite et pour fait internationalement licite devient dès lors, d'une part, la violation du droit international et, d'autre part, le risque.
} 


\subsection{Les sources du droit de la responsabilité internationale}

Il existe un régime de droit international général de la responsabilité internationale qui s'applique par défaut en l'absence de règles spéciales.

Les sources des règles générales applicables à la responsabilité internationale de l'État sont avant tout coutumières. La délimitation de ces règles coutumières des principes généraux de droit, qui en sont une autre source non-écrite, peut être difficile en pratique, toutefois. En effet, le droit de la responsabilité internationale puise une grande partie de son inspiration dans les principes issus des traditions nationales de la responsabilité civile ${ }^{13}$. En outre, c'est surtout grâce à la jurisprudence internationale, et notamment celle de la Cour internationale de justice (CIJ), que l'identification et l'interprétation de ces deux sources de règles non-écrites se fait ${ }^{14}$, ce qui rend la différence entre coutume et principes généraux encore plus difficile à faire.

En 2001, la Commission du droit international (CDI) des Nations unies a codifié les règles coutumières relatives à la responsabilité de l'État pour fait internationalement illicite dans une série d'articles: les ARSIWA. Elle a fait de même, en reprenant largement le même modèle que celui des ARSIWA, pour les règles relatives à la responsabilité des OI pour fait internationalement illicite et a adopté les DARIO en 2011. Alors que la valeur coutumière des premiers articles est incontestée, qu'elle ait précédé leur codification ou s'en soit ensuivie grâce à leur pratique jurisprudentielle notamment, tel n'est pas le cas de celle des DARIO. Ces derniers sont d'ailleurs encore à l'état de projet et n'ont pas fait l'objet d'une résolution de l'Assemblée générale des Nations unies. Les raisons de ce peu de reconnaissance sont que les règles codifiées/ créées par les DARIO n'étaient pas, pour la plupart, coutumières avant la codification, et n'ont pas été pratiquées depuis. La raison en est qu'il existe très peu d'obligations primaires des OI en droit international et donc d'obligations à violer, d'une part, et de tribunaux (nationaux ou internationaux) compétents (en soi ou pour des raisons d'immunités juridictionnelles) pour faire répondre les OI de la violation de ces éven-

13. Cf. A. Pellet, "The definition of responsibility in international law», in J. Crawford, A. Pellet, et S. Olleson (dir.), The Law of International Responsibility, Oxford, Oxford University Press, 2010, p. 3-16, p. 12-15.

14. Cf. C.J. Tams, «Law-making in complex processes: The World Court and the modern law of state responsibility", in C. Chinkin et F. Beytens (dir.), Sovereignty, Statehood and State Responsibility: Essays in Honour of James Crawford, Cambridge, Cambridge University Press, 2015, p. 287-306. Cf. par exemple, en matière de responsabilité solidaire: CIJ, Affaire du Détroit de Corfou, arrêt du 9 avril 1949; CIJ, Recueil 1949, p. 4; CIJ, Certaines terres à phosphates à Nauru (Nauru c. Australie), exceptions préliminaires, arrêt du 26 juin 1992; CIJ, Recueil 1992, p. 240 ; CIJ, Plates-formes pétrolières (République islamique d'Iran c. États-Unis d'Amérique), arrêt du 6 novembre 2003; CIJ, Recueil 2003, p. 161. 
tuelles obligations, d'autre part. La codification des DARIO elle-même a d'ailleurs été contestée pour toutes ces raisons-là ${ }^{15}$. C'est aussi parce que, si les ARSIWA ont été beaucoup critiqués pour s'être inspirés de catégories du droit privé national appliquées aux acteurs publics que sont les États, la reprise de ces catégories déjà hybrides et leur transposition aux OI est encore plus douteuse, alors même que la nature de leur personnalité est encore incertaine et que leur imbrication avec les États et leurs organes est très forte. S'il l'on peut comprendre qu'un sujet d'obligations juridiques doive pouvoir être tenu responsable de la violation de ses obligations ${ }^{16}$, le régime de cette responsabilité doit néanmoins refléter la nature du sujet en cause, des obligations qui le lient et de ses relations avec d'autres sujets responsables ${ }^{17}$. J'y reviendrai plus tard dans cette contribution.

En vertu du principe de la lex specialis, il existe diverses règles spéciales applicables à la responsabilité internationale de l'État et des OI (art. 55 ARSIWA, art. 64 et 67 DARIO).

Certaines sont issues de traités internationaux spéciaux, comme la Convention de 1972 sur la responsabilité internationale pour les dommages causés par des objets spatiaux ${ }^{18}$, la Convention de 1982 sur le droit de la $\operatorname{mer}^{19}$ ou encore les traités internationaux que l'UE et ses États membres concluent avec des États tiers ${ }^{20}$. On mentionnera aussi les règles internes à chaque OI dans la mesure où une OI peut prévoir un régime spécial de responsabilité internationale (art. 64 DARIO). C'est le cas notamment du régime de responsabilité financière (dans ses relations aux États tiers et aux autres OI) propre à l'UE dans le domaine des investissements internationaux ${ }^{21}$.

15. Cf. J. d'Aspremont «The articles on the responsibility of international organizations: Magnifying the fissures in the law of international responsibility ", International Organizations Law Review, vol. $9, \mathrm{n}^{\circ} 1,2012$, p. 15-28.

16. Cf. CIJ, Différend relatif à l'immunité de juridiction d'un rapporteur spécial de la Commission des droits de l'homme, avis consultatif du 29 avril 1999, CIJ, Recueil 1999, p. 62, § 66.

17. Voir également A.N. Pronto, "Reflections on the scope of application of the articles on the responsibility of international organizations ", in M. Ragazzi (dir.), Responsibility of International Organizations: Essays in Memory of Sir Ian Brownlie, Leyde, Martinus Nijhoff, 2013, p. 147-158.

18. Convention sur la responsabilité internationale pour les dommages causés par des objets spatiaux, Washington, Moscou et Londres, 29 mars 1982, UNTC 13810.

19. Convention des Nations unies sur le droit de la mer, Montego Bay, 10 décembre 1982, UNTC 31363.

20. Cf. la déclaration de compétence faite par la Communauté européenne en vertu de l'article 5, paragraphe 1, de l'annexe IX de la Convention de 1982 sur le droit de la mer; Tribunal international du droit de la mer, Affaire n² ${ }^{\circ}$, Avis consultatif du 2 avril 2015, $\$ 168$ et 172-174. Voir aussi P.J. Kuijper, «Attribution - responsibility - remedy. Some comments on the EU in different international regimes", Revue belge de droit international, vol. 46, $\mathrm{n}^{\circ}$ 1, 2013, p. 57-77.

21. Cf. par exemple les articles 3 et 9 du Règlement (UE) n 912/2014 du Parlement européen et du Conseil du 23 juillet 2014 établissant un cadre pour la gestion de la responsabilité financière liée aux tribunaux de règlement des différends entre investisseurs et États mis en place par les accords internationaux auxquels l'Union européenne est partie, JO L 257 du 28 août 2014, p. 121-134. 


\subsection{Les sujets de la responsabilité internationale}

Les sujets responsables de violation du droit international et ceux envers qui ils en sont responsables peuvent être des États et/ou des OI, même si la deuxième catégorie peut inclure des personnes privées, voire d'autres entités de droit international.

Les sujets responsables, premièrement. Les ARSIWA et DARIO ont codifié deux régimes de responsabilité internationale applicables selon que le responsable est un État ou une OI. Ces deux régimes sont concurrents dans la mesure où ils peuvent s'appliquer parallèlement si un État et une OI sont responsables ensemble (art. 57 ARSIWA; art. 65 DARIO). En fait, du fait de l'imbrication entre une OI et ses États membres, les DARIO s'appliquent aussi à la responsabilité des États qui sont liés au fait internationalement illicite d'une OI (art. 1[2] DARIO). Leurs régimes sont d'ailleurs même parfois entrecroisés et organisent le cumul des responsabilités. Ainsi, les DARIO contiennent certaines règles relatives à l'attribution de comportement ou de responsabilité des États aux OI (art. 14-19 DARIO) et vice-versa (art. 58-63 DARIO).

Il faut souligner que la responsabilité de droit international distincte des individus naît en marge des ARSIWA et DARIO (art. 58 ARSIWA; art. 66 DARIO). Il existe en effet certaines règles de droit international relatives à la responsabilité des particuliers, même si elles se limitent pour l'heure à la responsabilité pénale en vertu du droit international pénal. Ces règles peuvent dès lors s'appliquer parallèlement à la responsabilité des États et/ ou des OI. Par contre, la responsabilité des individus n'est pas soumise aux règles des ARSIWA et DARIO.

Les sujets lésés, deuxièmement. Les sujets envers qui les États et/ou les OI sont responsables dans les ARSIWA et DARIO sont les États et/ ou les OI (art. 33 ARSIWA; 33 DARIO). Cela n'exclut pas cependant les droits d'autres entités, et notamment des personnes privées (art. 33[2] ARSIWA; art. 33[2] DARIO). Le régime de la responsabilité des États pour la violation du droit international des droits de l'homme, qui est un régime de responsabilité envers les personnes privées, est d'ailleurs calqué sur le régime général de responsabilité internationale de l'État (et les ARSIWA ${ }^{22}$ ).

22. Cf. par exemple CourEDH, Banković et autres c. Belgique et autres, décision du 12 décembre 2001, Rec. 2001-XII, ECLI:CE:ECHR:2001:1212DEC005220799, §57. Pour une discussion de l'étendue de sa fidélité aux ARSIWA, cependant, voir par exemple J. Crawford et A. Keene, «The structure of state responsibility under the European Convention on Human Rights », in J. Motoc et A. von Aaken (dir.), The ECHR and General International Law, Oxford, op.cit. 


\subsection{Les conditions de la responsabilité internationale}

Selon les articles 1 ARSIWA et 3 DARIO, c'est le fait internationalement illicite qui engage la responsabilité internationale de l'État et/ou de l'OI. C'est le cas lorsque trois conditions sont remplies (art. 2 ARSIWA; art. 4 DARIO).

Premièrement, la violation d'une obligation primaire de droit international (art. 12 sq. ARSIWA; art. 10 sq. DARIO). Cette violation peut survenir par action ou par omission. L'obligation violée doit exister avant la violation, mais peut être de tout type (par ex. positive ou négative). Il faut noter que la distinction entre les obligations primaires qui sont violées et les obligations secondaires qui surgissent de cette violation et forment le contenu de la responsabilité internationale n'est pas aisée en pratique. Ainsi, certaines obligations (primaires) de diligence ou de non-assistance découlent du régime d'attribution du comportement illicite et donc du régime secondaire de la responsabilité internationale.

Deuxièmement, l'attribution du comportement constituant une violation à un État et/ou à une OI. La violation du droit international ou le fait internationalement illicite doit en effet pouvoir être attribué ou imputé à un État et/ou à une OI. Cela peut se faire soit par l'attribution (directe) du comportement d'organes, ou de particuliers, plus généralement, à l'État / l'OI (art. 4 sq. ARSIWA; art. 6 sq. DARIO), soit par l'attribution (indirecte) de la responsabilité d'un État / d'une OI à un autre État / à une autre OI (art. 16 sq. ARSIWA; art. 14 sq.; et 58 sq. DARIO).

L'attribution de comportement/responsabilité peut donc se faire à un seul, mais aussi à plusieurs États/OI responsables selon les $\operatorname{cas}^{23}$. C'est, tout d'abord, l'attribution de responsabilité qui va contribuer à multiplier les sujets responsables, puisque la responsabilité attribuée doit elle-même l'avoir été par attribution de comportement à un premier État/OI ${ }^{24}$. Ce n'est pas nécessairement le cas, cependant. Ainsi, dans le cas du contournement d'obligations (art. 17 et 61 DARIO), il peut n'y avoir qu'un seul sujet responsable: celui auquel la responsabilité est attribuée. En outre, il peut aussi y avoir une multiplication des États/OI responsables par le biais de l'attribution conjointe de comportements lorsque le contrôle sur les organes d'un État ou d'une OI est conjoint à celui d'autres États ou OI sur ces mêmes organes (art. 6 ARSIWA; art. 7 DARIO $^{25}$ ). Enfin, différents États peuvent se voir attribuer des comportements indépendamment les uns des autres et répondre du même préjudice ainsi causé.

23. Cf. J. Crawford, op. cit., p. 333 sq.

24. Cf. J.D. Fry, «Attribution of responsibility», in A. Nollkaemper et I. Plakokefalos (dir.), Principles of Shared Responsibility in International Law op. cit., p.98-133.

25. Cf. F. Messineo, «Attribution of Conduct», in A. Nollkaemper et I. Plakokefalos (dir.), Principles of Shared Responsibility in International Law, op. cit., p. 60-97. 
L'attribution de comportement, tout d'abord, permet d'attribuer le comportement de leurs organes aux sujets de responsabilité, et donc soit le comportement d'organes, ou plus généralement de particuliers, à un État / à une OI, soit celui des organes ou agents d'un État / d'une OI à une OI (art. 4-11 ARSIWA; art. 6-9 DARIO; y compris pour des actes ultra vires [art. 7 ARSIWA; art. 8 DARIO]).

Parmi les types d'attribution, on peut distinguer l'attribution du comportement des organes de jure de l'État / de l'OI (art. 4 ARSIWA; art. 6 DARIO); de personnes autorisées à exercer des prérogatives de puissance publique à l'État (art. 5 ARSIWA); d'organes mis à la disposition de l'État / de l'OI par un autre État / une autre OI (art. 6 ARSIWA; art. 7 DARIO); de personnes sous le contrôle effectif de l'État (organes de facto) (art. 8 ARSIWA); de personnes agissant selon la gestion d'affaires sans mandat pour l'État (art. 9 ARSIWA); ou d'un mouvement insurrectionnel lorsque ce dernier devient le nouveau gouvernement de l'État ou d'un nouvel État (art. 10 ARSIWA). On mentionnera encore la reconnaissance de comportement par l'État / l'OI (art. 11 ARSIWA; art. 9 DARIO). Il faut remarquer que toutes ces catégories d'attribution de comportement à l'État n'existent pas pour les OI; le fondement d'attribution de responsabilité dans les DARIO est avant tout lié aux fonctions des OI et donc de ceux qui agissent pour elles dans le cadre de ses fonctions. L'addition d'une référence aux «agents» des OI en sus de leurs «organes» constitue néanmoins une base d'ouverture potentielle en matière d'attribution de responsabilité.

L'attribution de responsabilité, ensuite, permet d'attribuer la responsabilité d'un État / d'une OI à un autre État / une autre OI (art. 16-19 ARSIWA; art. 14-19 DARIO; art. 58-62 DARIO).

Parmi les fondements d'attribution, on peut distinguer l'assistance/complicité (art. 16 ARSIWA; art. 14 et 58 DARIO); le contrôle ou les directives (art. 17 ARSIWA; art. 15 et 59 DARIO); la contrainte (art. 18 ARSIWA; art. 16 et 60 DARIO); et le contournement d'obligations (art. 17 et $61^{26}$ DARIO). À noter que le contournement d'obligations comme fondement d'attribution de responsabilité est propre aux OI. C'est un mode d'attribution lié à l'abus potentiel de la personnalité des OI par leurs États membres, et vice-versa. Il ressemble d'ailleurs plus à une obligation primaire de diligence due ou raisonnable ex ante qu'à un fondement d'attribution de responsabilité ex post. J'y reviendrai plus tard dans cette contribution. Il faut souligner en outre la condition de l'existence d'une obligation internationale de l'État /

26. Art. 61 DARIO: «Contournement des obligations internationales d'un État membre d'une organisation internationale. 1. Un État membre d'une organisation internationale engage sa responsabilité internationale si, en se prévalant du fait que l'organisation est compétente relativement à l'objet d'une des obligations internationales de cet État, il contourne cette obligation en amenant l'organisation à commettre un fait qui, s'il avait été commis par cet État, aurait constitué une violation de cette obligation. 2. Le paragraphe 1 s'applique que le fait en question soit ou non internationalement illicite pour l'organisation internationale». 
de l'OI assisté(e)/dirigé(e)/contraint(e) pour que leur responsabilité puisse être attribuée à un autre État / une autre OI, bien que cette condition n'existe pas pour le cas du contournement d'obligations (art. 17 et 61 DARIO). Cela est particulièrement important en cas de violation du droit international des droits de l'homme puisque ce dernier ne lie pas les OI, mais que le droit des OI peut amener à en causer la violation sans que les États n'aient de marge d'appréciation et donc sans possibilité que ces violations leur soient attribuées directement.

Troisièmement, l'absence de circonstances excluant l'illicéité (art. 20 sq. ARSIWA; art. 20 sq. DARIO). Il s'agit du consentement, de la légitime défense, des contre-mesures, de la force majeure, de la détresse et de l'état de nécessité.

Finalement, il faut remarquer l'absence d'exigence d'un préjudice (matériel ou moral) parmi les conditions de la responsabilité internationale des États/OI. Le préjudice n'est pas une condition formelle de la responsabilité internationale, la violation objective du droit international étant une forme de «préjudice légal» suffisante. C’est une différence importante entre le régime de la responsabilité de droit civil national et la responsabilité internationale.

Le préjudice demeure néanmoins un élément incontournable du régime de la responsabilité internationale. C'est en effet ce que la violation du droit international a causé et ce qui justifie fondamentalement la responsabilité. C'est l'existence d'un préjudice qui donne droit à une réparation et permet de faire un lien entre le fait internationalement illicite et l'État /l'OI lésé(e) par référence au tort causé ${ }^{27}$. C'est aussi le préjudice qui permet de fixer le moment de la violation dans le temps, puis l'objet de la réparation. Comme nous le verrons, c'est le fait d'avoir causé un même préjudice qui lie les différents États/OI responsables en cas de pluralité d'États / d'OI responsables, et non l'identité des obligations violées ou du fait illicite. C'est d'ailleurs aussi le préjudice, et le lien de causalité qui relie le fait illicite au préjudice, qui permettent d'allouer les responsabilités entre États/OI impliqués et les réparations. On pourrait dès lors voir dans cette absence de prise en compte du préjudice dans les ARSIWA/DARIO une explication à la négligence de la question de la responsabilité plurale et de la solidarité des responsables: la distribution des responsabilités, réparations et recours internes éventuels exige en effet de la clarté quant aux questions de causalité en droit international de la responsabilité et tel est loin d'être le cas actuellement.

Par ailleurs, on remarquera aussi l'absence d'éléments subjectifs, comme l'intention ou la faute, parmi les conditions de la responsabilité internationale. La responsabilité internationale est objective et déclenchée par la

27. Cf. P. d'Argent, op.cit., p. 220. 
simple violation d'une obligation internationale. C'est une autre différence importante entre le régime de la responsabilité de droit civil national et la responsabilité internationale.

À nouveau, ces éléments resurgissent tout de même à différents endroits du régime de la responsabilité internationale. On mentionnera, par exemple, le rôle de la diligence dans l'obligation de diligence due en matière d'attribution du comportement d'organes de jure, ou celui de l'intention en matière de complicité ou du contournement d'obligations. Ces éléments subjectifs, et notamment la diligence, peuvent ensuite avoir leur importance dans la répartition des responsabilités plurales, comme nous le verrons d'ailleurs ${ }^{28}$. À nouveau, au vu de leur rôle dans ce contexte, on peut voir dans l'absence de prise en compte des éléments subjectifs dans les ARSIWA/DARIO une explication de la négligence de la question de la responsabilité plurale et de la solidarité des responsables dans ces articles.

\subsection{Le contenu de la responsabilité internationale}

Le contenu de la responsabilité internationale comprend les obligations secondaires, ou, en bref, les « responsabilités », engagées par le fait internationalement illicite (art. 28 sq. ARSIWA; art. 28 sq. DARIO).

Il s'agit d'obligations de cessation, si la violation est durable et que l'obligation violée est continue; de non-répétition, si l'obligation est continue et que les circonstances l'exigent; et, enfin, de réparation, s'il y a un préjudice. Cette dernière peut prendre différentes formes (en relation de subsidiarité les unes aux autres; art. 34 sq. ARSIWA; art. 34 sq. DARIO): tout d'abord, la restitution de la situation antérieure à la violation (restitutio in integrum); lorsque ce n'est pas possible, l'indemnisation du préjudice causé; et, lorsque ni l'une ni l'autre ne sont faisables, la satisfaction (par ex. des excuses).

C'est sur les obligations secondaires de réparation, et avant tout sur l'indemnisation, que cette contribution se concentrera. En effet, les autres obligations secondaires qui forment le contenu de la responsabilité ne peuvent guère être dues par une pluralité d'États et/ou d'OI. Seul un État ou une OI parmi la pluralité d'entités responsables sera en général en mesure d'assurer la restitution (vers l'état antérieur à la violation) ou la satisfaction (non-financière ${ }^{29}$ ).

\footnotetext{
28. Sur l'obligation de diligence comme moyen de créer une responsabilité solidaire, voir aussi l'introduction d'Alain Supiot à ce volume. Notons que la violation d'une obligation de diligence constitue un fait internationalement illicite en droit de la responsabilité internationale et n'est donc pas le fondement d'une responsabilité causale ou sans faute, contrairement à ce qui vaut en droit interne. 29. Cf. S. Besson, 2007, op. cit, p. 11; P. d'Argent, op. cit., p. 215 sq. et p. 236 sq.
} 


\subsection{Un point terminologique}

Quelques clarifications terminologiques peuvent désormais être proposées sur la base de cette présentation du régime de la responsabilité internationale. Lorsque plusieurs États/OI sont responsables du même fait illicite (par attribution de comportement ou de responsabilité), on parle de "concours » ou de "pluralité de responsabilités». Les termes utilisés varient beaucoup, cependant, y compris entre le français et l'anglais ${ }^{30}$, et il convient d'être prudent $^{31}$.

La responsabilité "plurale» (plural or multiple responsibility), premièrement, est la responsabilité d'une pluralité d'États et/ou d'OI. Elle est "partagée » (shared responsibility) lorsqu'il s'agit d'une responsabilité d'une pluralité d'États et/ou d'OI pour le même préjudice. Elle est «concurrente» (concurring responsibility), lorsqu'il s'agit de la responsabilité d'une pluralité d'États et/ou d'OI pour le même préjudice causé par plusieurs faits illicites distincts. Par contre, elle est «conjointe» (joint responsibility) lorsqu'il s'agit d'une responsabilité d'une pluralité d'États et/ou d'OI pour le même préjudice, causé par le même fait illicite. Il doit toutefois s'agir d'un seul acte conjoint, et non pas d'une série d'actes distincts mais identiques. Enfin, la responsabilité est dite "solidaire" (joint and several responsibility) lorsqu'il s'agit d'une responsabilité d'une pluralité d'États et/ou d'OI pour le même préjudice et du fait du même fait illicite, et qui oblige de ce fait chacun à réparer l'entier du préjudice causé par leur fait illicite.

En somme, la responsabilité "solidaire» est une forme de responsabilité «partagée» et «conjointe» qui est elle-même une forme de responsabilité «plurale».

\section{La répartition des responsabilités plurales en droit international : responsabilités divise et solidaire}

Lorsque plusieurs États et/ou OI ont causé le même préjudice, la question de la répartition, de l'allocation ou de la distribution de leurs responsabilités se pose. C'est sur cette base, en effet, que la répartition des obligations de réparation pourra ensuite se faire. Malgré le nombre croissant de cas de responsabilités partagées en pratique, les questions juridiques que pose la répartition des responsabilités sont nouvelles et attendent encore des réponses.

30. En fait, les variations valent aussi entre traditions juridiques (y compris dans la même langue). 31. Voir par exemple A. Nollkaemper et D. Jacobs, op. cit., p. 366-368; J. Crawford, op. cit., p. 333 sq. 


\subsection{Le principe: la responsabilité individuelle et divise}

Le principe est que la responsabilité internationale est (i) indépendante ou distincte, et (ii) divise (art. 42 et 47[1] ARSIWA; art. 43 et 48[1] DARIO). Lorsque plusieurs États et/ou OI ont causé le même préjudice, par conséquent, chaque État et/ou OI responsable peut être recherché(e) individuellement et séparément (et non pas conjointement avec d'autres) (i), mais ne doit répondre de la violation de ses obligations internationales qu'à hauteur de sa contribution au préjudice causé (et non pas de l'entier de ce dernier) (ii).

La conséquence de la responsabilité divise est que les responsabilités de chaque État et/ou OI doivent être divisées, puis allouées, distribuées ou réparties en fonction de la contribution de chacun au préjudice causé. Se pose dès lors la question des critères aptes à déterminer le degré de cette contribution.

Le premier critère est la causalité. Comme je l'ai expliqué, cependant, cette question, pourtant si centrale pour la réparation et sa répartition, n’a encore été que peu abordée en droit international de la responsabilité ${ }^{32}$. Lorsqu'elle l'est, c'est souvent de manière superficielle au vu de la complexité des cas, notamment lorsqu'il y a une pluralité de faits illicites, une combinaison de responsabilités diverses par attribution de comportement et de responsabilité, et la violation d'une obligation de diligence ${ }^{33}$.

Sur la base de la pratique nationale en la matière, on peut distinguer quatre types de causalité potentielle applicables en cas de pluralité d'États et/ ou d'OI impliqués dans un même préjudice ${ }^{34}$. La causalité peut, premièrement, être exclusive. Dans ces cas, malgré les apparences, il n'y a qu'un seul fait illicite qui a causé le préjudice. La répartition des responsabilités peut donc se faire de manière individuelle au seul État / à la seule OI responsable. La causalité peut, deuxièmement, être parallèle. Dans cette hypothèse, chaque fait illicite aurait pu conduire au même préjudice indépendamment des autres. La répartition individuelle à chaque État/OI responsable est donc possible. Troisièmement, la causalité peut aussi être complémentaire. Dans ce cas, chaque fait illicite aurait pu conduire à un préjudice indépendamment des autres, mais pas au préjudice complet. Ce dernier est un agrégat de tous les

\footnotetext{
32. Cf. B. Stern, «The Obligation to Make Reparation», in J. Crawford, A. Pellet et S. Olleson (dir.), The Law of International Responsibility, Oxford, Oxford University Press, 2010, p.563-571; A. Gattini, «Breach of International Obligations », in A. Nollkaemper et I. Plakokefalos (dir.), Principles of Shared Responsibility in International Law, op. cit., p. 25-59, en particulier p. 28-31. Selon P. d'Argent, op.cit., p. 223 sq., la raison en est la caractéristique éminemment élective, et donc arbitraire, de la causalité en droit (de la responsabilité).

33. Cf. par exemple l'analyse maladroite de la CIJ, Application de la convention pour la prévention et la répression du crime de génocide (Bosnie-Herzégovine c. Serbie-et-Monténégro), arrêt du 26 février 2007, CIJ, Recueil 2007, p. 43, \$460-463.

34. Cf. P.d'Argent, op. cit., p. 223 sq.
} 
préjudices distincts ainsi causés. La répartition peut alors se faire de manière proportionnelle à la contribution de chaque État/OI responsable au préjudice causé. Finalement, la causalité peut être cumulative. Dans ce cas, chaque fait illicite pris séparément n'aurait pas pu conduire à un préjudice et devait avoir lieu avec les autres pour produire ce préjudice qui peut être considéré comme indivisible. Dans ces cas, la répartition individuelle et proportionnelle des responsabilités qui reviennent à chaque État/OI responsable est impossible sur le plan de la causalité stricte. Parmi les moyens de répartir les responsabilités en cas de causalité cumulative, on peut mentionner la sélection d'une cause «adéquate» ou reconstruite (par exemple la plus récente) ou encore opter pour l'«équivalence» des causes et donc diviser la responsabilité par le nombre d'États / d'OI responsables ${ }^{35}$.

\subsection{L'exception : la responsabilité individuelle et indivise}

Dans ces conditions de causalité complexe, et notamment de causalité cumulative, une responsabilité individuelle ou distincte, mais solidaire ou indivise (joint and several responsibility) serait plus juste. Ce principe n'est pas inconnu en droit de la responsabilité internationale, mais il est fortement limité dans son régime et peu appliqué.

\subsubsection{Le régime de la responsabilité solidaire internationale}

En cas de pluralité d'États et/ou d'OI impliqués dans un même préjudice, le droit international général de la responsabilité connaît, comme le droit national de la responsabilité civile, le principe de la responsabilité solidaire (art. 47[1] et [2] ARSIWA; art. 48[1] et [3] DARIO a contrario ${ }^{36}$ ). Cette institution permet au sujet lésé (État, OI ou particulier) (i) de ne rechercher

\footnotetext{
35. Cf. P. d'Argent, op. cit., p. 229-232.

36. À noter que certains auteurs (par exemple J. Crawford, op. cit., p. 333 sq. et p. 355 ; CDI, «Projet d'articles sur la responsabilité de l'État pour fait internationalement illicite et commentaires y relatifs ", Annuaire de la Commission du droit international, vol. II, nº 2, 2001, p. 31-154, en particulier p. 133 sq. : commentaire de l'art. 47, \$ 6), considèrent que les articles 47(1) ARSIWA / 48(1) DARIO n'excluent pas la responsabilité solidaire (ce qui découle de l'article 47[2] ARSIWA / art. 48[3] DARIO), mais n'en font pas une solution par défaut en présence d'un même fait illicite, alors que d'autres auteurs (par exemple J.E. Noyes et B.D. Smith, op. cit., p. 249 sq.; CIJ, Plates-formes pétrolières [République islamique d'Iran c. États-Unis d'Amérique], opinion individuelle de M. le Juge Simma, CIJ, Recueil 2003, p. 166, \$63-83; S. Besson, op. cit., 2007, p. 21-22; A. Orakhelashvili, op. cit., p. 647-665; P. d'Argent, p. 240-241) considèrent que la solidarité est le régime par défaut des articles 47(1) ARSIWA / 48(2) DARIO, au risque sinon de vider les prescriptions des articles 47(2) ARSIWA / 48(3) DARIO de leur sens. Le débat semble aujourd'hui se réduire largement à un débat d'étiquettes.
} 
qu'un seul des États et/ou OI responsables (la responsabilité demeure donc individuelle ou indépendante), mais (ii) d'obtenir réparation de l'ensemble de son préjudice de ce dernier (la responsabilité devient indivise).

Il faut remarquer qu'il n'y a pas de responsabilité conjointe (non-individuelle) en droit international de la responsabilité, même si certains régimes spéciaux peuvent le prévoir. Chaque État/OI responsable peut donc toujours être recherché(e) individuellement même en cas de responsabilité solidaire ${ }^{37}$. D'ailleurs, le principe de la tierce partie indispensable, qui limitait cette possibilité sur le plan procédural ${ }^{38}$, est désormais interprété largement ${ }^{39}$.

Il ne faut pas confondre la responsabilité solidaire avec la responsabilité subsidiaire qui existe en droit de la responsabilité des OI (art. 48[2] DARIO). Selon ce principe, l'État / l'OI responsable subsidiairement répond dès que le responsable primaire ou principal ne peut pas répondre. Cela s'applique dans certains cas rares d'attribution de responsabilité de droit international général, comme la reconnaissance de responsabilité (par exemple l'art. 62 [2] DARIO), mais plus fréquemment en matière de droit international spécial de la responsabilité. Bien sûr, la responsabilité subsidiaire peut aussi être solidaire. Elle ne l'est pas forcément, cependant, et, à l'inverse, toute responsabilité solidaire n'implique pas de subsidiarité.

Les conditions et principes de mise en œuvre de la responsabilité solidaire en droit international de la responsabilité sont très stricts, néanmoins.

Tout d'abord, la condition d'application de la responsabilité solidaire est que le préjudice causé par les États et/ou OI responsables l'ait été par un même fait illicite (art. 47[1] ARSIWA; art. 48[1] DARIO). Il doit s'agir d'un seul et même fait conjoint, et non pas d'une série de faits distincts mais identiques ${ }^{40}$.

Il y a un même fait illicite ou un fait illicite conjoint notamment dans deux types de circonstances. Premièrement, c'est le cas lorsque les États et/ ou OI exercent un contrôle effectif sur les mêmes organes (individus/États) et que le comportement de ces organes peut leur être attribué conjointement (art. 6 ARSIWA; art. 7 DARIO). Une deuxième hypothèse est celle de l'attribution de comportement à l'un des États/OI responsables et de l'attribution de la responsabilité pour ce même comportement à un autre en raison du contrôle, de la contrainte ou des directives qu'il exerce sur le premier (art. 17-18 ARSIWA; art. 15-16/59-60 DARIO). À noter qu'en cas d'attribution de responsabilité par contrôle (art. 59 DARIO), plus les États/OI sont contrôlés, moins ils seront en mesure d'être responsables

37. Cf. CIJ, Détroit de Corfou, $\$ 22-23$; CIJ, Phosphates de Nauru, $\$ 258-262$.

38. Cf. CIJ, Affaire de l'or monétaire pris à Rome en 1943 (question préliminaire), arrêt du 15 juin 1954, CIJ, Recueil 1954, p. 19, \$32. Nuancé dans CIJ, Timor Oriental (Portugal c. Australie), arrêt du 30 juin 1995, CIJ, Recueil 1995, p. 90, \$102-105.

39. Cf. CIJ, Phosphates de Nauru, $\$ 258-262$; CIJ, Plates-formes pétrolières.

40. Cf. P.d'Argent, p. 213 et 241-244 sur la «coperpétration». 
en leur nom propre. Il faut aussi rappeler qu'en cas de contournement d'obligations d'une OI par un État membre ou vice-versa (art. 17 et 61 DARIO), il se peut qu'il n'y ait qu'un seul sujet responsable (celui qui contourne ses obligations en instrumentalisant l'autre), mais aussi deux sujets responsables si le fait illicite du sujet qui instrumentalise remplit les conditions pour donner lieu à une responsabilité première du sujet instrumentalisé. L'exemple qu'on peut donner d'un fait illicite conjoint est la pollution d'une rivière par deux États riverains parce que l'autorité de régulation des émissions commune aux deux États a failli à sa mission ${ }^{41}$. Une série de faits illicites distincts mais identiques serait l'agression armée d'un État par une coalition d'États lors d'un bombardement conjoint ${ }^{42}$.

Par contraste, il y a plusieurs faits illicites distincts, et aucune responsabilité solidaire, même s'ils sont identiques, lorsque chacun des États/OI responsables l'est par attribution de comportements distincts. C'est aussi le cas lorsque l'un d'entre eux est l'auteur principal et l'autre / les autres l'est / le sont par attribution de responsabilité du fait de l'assistance/aide qu'il/ils a/ont fournie au premier, ce qui constitue un fait illicite distinct (complicité; article 16 ARSIWA; article 14/58 DARIO) ${ }^{43}$. L'exemple qu'on peut donner est celui de l'extradition d'une personne par un État vers un autre en vue de sa torture dans le deuxième État, le préjudice causé par les deux faits illicites distincts étant la torture ${ }^{44}$. Un autre exemple est celui où différents États ont contribué à l'explosion d'un navire étranger, l'un par le mouillage de mines et l'autre pour ne pas les avoir signalées alors qu'elles se trouvaient dans ses eaux territoriales ${ }^{45}$.

Ensuite, les principes de mise en ouvre de la responsabilité solidaire limitent aussi son applicabilité en pratique. Ils sont au nombre de deux (art. 47[2] ARSIWA; art. 48[3] DARIO).

Premièrement, l'interdiction de l'enrichissement illégitime. Le lésé ne doit pas recevoir une réparation supérieure au préjudice subi. Par conséquent, le cumul d'actions est interdit: la réparation par un des États/OI responsables libère les autres de leurs responsabilités externes envers le sujet lésé. Cela vaut jusqu'au moment des recours internes, bien sûr. Deuxièmement, l'existence de recours internes. L'État/OI responsable qui a porté la responsabilité entière du préjudice doit pouvoir se retourner contre les autres responsables.

\footnotetext{
41. Cf. dans le même sens: CIJ, Phosphates de Nauru.

42. Cf. dans le même sens: CIJ, Plates-formes pétrolières.

43. Cf. H. Aust, Complicity and the Law of State Responsibility, Cambridge, Cambridge University Press, 2013, p. 487.

44. Cf. dans le même sens: CourEDH, Soering c. Royaume-Uni, arrêt du 7 juillet 1989, Séries A 161, ECLI:CE:ECHR:1989:0707JUD001403888.

45. Cf. dans le même sens: CIJ, Détroit de Corfou, \$22-23.
} 


\subsubsection{Les limites de l'applicabilité et de l'application de la responsabilité solidaire internationale}

Le principe de responsabilité solidaire ne trouve qu'une application limitée en droit international, et ce, tant pour des raisons qui lui sont propres que pour des raisons liées à l'état de l'ordre juridique international ${ }^{46}$.

Tout d'abord, le champ d'application du régime de responsabilité solidaire en droit de la responsabilité internationale est limité de manière inhérente, comme nous l'avons vu, par des conditions d'applicabilité strictes.

La raison en est double. Premièrement, si les États/OI ont agi ensemble par un même fait illicite conjoint, il sera très difficile d'allouer / de répartir les responsabilités individuelles entre les sujets responsables. Dans ce genre de cas, en effet, la causalité sera cumulative la plupart du temps. Deuxièmement, si les États/OI ont agi ensemble par un même fait illicite conjoint et ont donc pu se coordonner pour agir ensemble, ils peuvent coordonner leur réaction et leurs responsabilités en réparant ensemble, puis en organisant ensuite les recours internes entre eux pour se répartir leurs responsabilités respectives.

A contrario, la crainte, dans les autres cas, est que les États/OI ne puissent pas se coordonner pour assurer les recours internes et éviter l'enrichissement illégitime de l'un ou de plusieurs d'entre eux, alors même qu'il est possible, et donc plus facile, de répartir les responsabilités individuelles entre eux. En effet, il manque encore sur le plan international un législateur centralisé pour fixer un régime de recours internes en cas de responsabilité solidaire ex ante. Il manque, en outre, des remèdes judiciaires effectifs pour fixer les responsabilités de chacun ex post et coordonner les recours internes entre eux. Il n'y a, en effet, la plupart du temps pas de tribunal international compétent - et s'il y en a, il y en a parfois trop - ou, en tout cas, pas de tribunal dont la juridiction serait obligatoire.

En somme, la solidarité limitée en matière de responsabilité internationale des États et/ou OI reflète l'état des relations internationales, et notamment le peu d'institutionnalisation qui les caractérise actuellement ${ }^{47}$.

Ensuite, même lorsqu'il est applicable, le principe de responsabilité solidaire n'est que rarement mis en œuvre en pratique. Il y a notamment

\footnotetext{
46. Certains auteurs y voient même le signe de l'absence de base coutumière des articles 47 ARSIWA / 48 DARIO et du principe de responsabilité solidaire en droit international (dans son acception limitée actuelle). Voir par exemple P. d'Argent, op. cit., p. 245 sq.

47. Cf. M. Koskenniemi, «Solidarity measures: state responsibility as a new international order?», British Yearbook of International Law, vol. 72, $\mathrm{n}^{\circ}$ 1, 2001, p. 337-356. Sur la solidarité comme principe général de droit international plus généralement, voir aussi $\mathrm{K}$. Wellens, «Revisiting solidarity as a (re-)emerging constitutional principle: some further reflections», in R. Wolfrum et C. Koijma (dir), Solidarity: A Structural Principle of International Law, Dodrecht, Springer, 2010, p. 3-38; S. Besson, "Community interests in international law-making - With a special emphasis on treaty interpretation and identification of customary international law», in E. Benvenisti et G. Nolte (dir.), Community Obligations in Contemporary International Law, 2018.
} 
très peu de jurisprudence apte à être discutée pour faire évoluer l'interprétation des articles 47 ARSIWA et 48 DARIO. Ce sont toujours les mêmes arrêts, désormais anciens ${ }^{48}$, qui sont cités pour confirmer la possibilité de la responsabilité solidaire. Aucun d'entre eux ne permet cependant d'emporter la conviction dans la mesure où certains semblent, selon les interprétations, favoriser la responsabilité divise ${ }^{49}$. Les travaux préparatoires des articles 47 ARSIWA / 48 DARIO ne sont d'aucun secours à cet égard, ces dispositions ayant été intégrées tardivement dans la codification et sans grandes discussions $s^{50}$.

Seuls certains régimes spéciaux de responsabilité en droit international ont expressément choisi d'appliquer la responsabilité solidaire et en ont par conséquent précisé les modalités ${ }^{51}$. C'est le cas, par exemple, des articles IV et V de la Convention de 1972 sur la responsabilité internationale pour les dommages causés par des objets spatiaux et de l'article 6 , annexe IX de la Convention de 1982 sur le droit de la mer ${ }^{52}$. C'est aussi le cas dans quelques-unes des règles internes mises en place par certaines OI ou comprises dans leurs accords mixtes ${ }^{53}$. On mentionnera, par exemple, les articles 3 et 9 du Règlement de l'UE relatif à la responsabilité financière en matière d'investissements ${ }^{54}$. La raison en est certainement qu'il s'agit là de régimes dans lesquels l'action collective à l'origine de violations éventuelles est coordonnée, d'une part, et que cette coordination ex ante de l'action pourrait donc être transposée à celle des responsabilités suite à ces violations, d'autre part.

En droit international des droits de l'homme, en revanche, ainsi que dans la plupart des autres régimes de droit international, la responsabilité solidaire ne trouve aucune application. Et cela alors même que plusieurs États sont recherchés en responsabilité ensemble devant le même tribunal

\footnotetext{
48. Cf. CIJ, Détroit de Corfou, $\$ 22-23$; CIJ, Phosphates de Nauru; CIJ, Plates-formes pétrolières. Voir, cependant, l'affaire pendante CIJ, Activités armées sur le territoire du Congo (République démocratique du Congo c. Ouganda), arrêt du 19 décembre 2005, CIJ, Recueil 2005, p. 168.

49. Cf. US Court of Claims, Anglo-Chinese Shipping Co Ltd v. United States, International Law Reports, vol. 22, 1955, p. 982-986; Cour permanente d'arbitrage, The Channel Tunnel Group Limited et France-Manche SA c. Secretary of State for Transport of the Government of the United Kingdom of Great Britain and Northern Ireland et Ministre de l'équipement, des transports, de l'aménagement du territoire, du tourisme et de la mer du Gouvernement de la République française, ('Eurotunnel'), sentence partielle du 30 janvier 2007.

50. Cf. J.B. Crawford, op. cit., p. 326-327.

51. Cf. S. Besson, op. cit., 2007, p. 18-19; P. d'Argent, op. cit., p. 245-246.

52. Voir sur l'UE et sa déclaration au sens des articles 5 et 6 de l'Annexe IX UNCLOS: Tribunal international du droit de la mer, Affaire $n^{\circ} 21, \$ 168$ et 172-174. Voir aussi P.J. Kuijper, op. cit.

53. Cf. par exemple l'article 3(7) du Projet révisé d'accord portant adhésion de l'Union européenne à la Convention de sauvegarde des droits de l'homme et des libertés fondamentales, Strasbourg, 10 juin 2013. Cf. M. Den Heijer et A. Nollkaemper, «A new framework for allocating international responsibility: the EU accession to the European Convention on Human Rights», SHARES Briefing Paper, 2014, www.sharesproject.nl (consulté le 28 août 2016).

54. Règlement (UE) n 912/2014 sur les investissements.
} 
international et sur la base du même instrument international de protection des droits de l'homme, d'une part, et que les conditions d'application de la responsabilité solidaire sont remplies, d'autre part.

On peut avancer les raisons suivantes pour expliquer pourquoi la responsabilité solidaire a été négligée par les praticiens du droit international à ce jour.

En droit international général, premièrement ${ }^{55}$. Il faut tout d'abord souligner le fait que des procédures judiciaires nationales/internationales ou de règlement des différends internationaux n'existent pas dans tous les cas, et que, même lorsqu'elles existent, elles peuvent encore se heurter à des problèmes d'immunité. Même si la responsabilité n'exige pas sa reconnaissance procédurale, la résolution d'un concours de responsabilités et la répartition des réparations la requièrent. Deuxièmement, même lorsque ces procédures existent, elles n'ont pas toujours un caractère obligatoire. Certaines responsabilités y sont soumises et d'autres non. Troisièmement, même lorsque ces procédures existent et sont obligatoires, leur juridiction est souvent limitée ratione personae ou materiae. Toutes les responsabilités en cause pour le même fait illicite ne peuvent donc pas être examinées en même temps. Quatrièmement, même lorsque ces procédures existent et sont obligatoires, on remarque l'absence de hiérarchie ou de cohérence entre elles (par ex. pas de litispendance / ne bis in idem). Les responsabilités respectives peuvent donc être établies indépendamment les unes des autres, ce qui permet d'éventuelles reconnaissances multiples de responsabilité, et encourage donc la séparabilité des responsabilités ${ }^{56}$. Cinquièmement, on mentionnera le caractère bilatéral du contentieux international et des procédures judiciaires internationales / de règlement des différends internationaux. Ces procédures sont donc peu à même de prendre en compte le concours de responsabilités d'autres États/OI que celui ou celle mis(e) en cause. Sixièmement, il faut rappeler le principe de la tierce partie indispensable, c'est-à-dire la condition procédurale selon laquelle une affaire qui met en cause un État ne peut pas être tranchée sans son consentement, ni, par conséquent, sans son implication dans la procédure ${ }^{57}$. Ce principe fait cependant l'objet d'interprétations larges désormais ${ }^{58}$.

\footnotetext{
55. Cf. S. Besson, op. cit., 2007, p.6-7.

56. Pour une critique, voir A. Nollkaemper, "Concerted adjudication in cases of shared responsibility», NYU Journal of International Law and Politics, vol. 46, n³, 2014, p. 809-847.

57. Cf. CIJ, Affaire de l'or monétaire, $\$ 32$. Nuancé dans CIJ, Timor-Oriental, $\$ 102-105$.

58. Cf. CIJ, Phosphates de Nauru, $\$ 258-262$; CIJ, Plates-formes pétrolières. Voir aussi en droit international des droits de l'homme, ComEDH, Hess c. Royaume-Uni, décision du 28 mai 1975, D.R. n ${ }^{\circ}$, p. 74, mais nuancé par CourEDH, M.S.S. c. Belgique et Grèce, arrêt du 21 janvier 2011, Rec. 2011-I, ECLI:CE:ECHR:2011:0121JUD003069609, \$342; CourEDH, Behrami et Behrami c. France et Saramati c. France, Allemagne et Norvège, décision du 2 mai 2007, ECLI:CE:ECHR:2007:0502DEC007141201, \$67.
} 
En droit international des droits de l'homme, deuxièmement ${ }^{59}$. Les raisons générales pour l'applicabilité limitée du principe de responsabilité solidaire en droit de la responsabilité internationale s'appliquent aussi dans ce contexte.

A priori, certes, on aurait attendu que ce soit moins le cas, et ce, du fait de certaines caractéristiques procédurales du droit international des droits de l'homme. On pensera notamment à l'existence de tribunaux internationaux en matière de droits de l'homme, dont la juridiction est obligatoire, comme la Cour européenne des droits de l'homme (CourEDH), mais aussi à la possibilité d'attraire plusieurs États en même temps devant la CourEDH ${ }^{60}$. D'ailleurs, la jurisprudence de la CourEDH comprend différents exemples de cas dans lesquels plusieurs États ont été poursuivis ensemble ${ }^{61}$ ou du moins mis en cause dans une affaire qui concernait un autre État ${ }^{62}$, voire même une OI à travers tous ses États membres ${ }^{63}$.

Il est d'autant plus intriguant dès lors que la pratique du droit international des droits de l'homme soit si réticente à recourir à la responsabilité solidaire. En effet, la plupart des arrêts pertinents de la CourEDH dans des affaires de concours de responsabilités traite les responsabilités plurales comme distinctes et répartit les responsabilités en fonction de la contribution de chacun au préjudice causé ${ }^{64}$. Et cela même lorsque la violation provient du même fait illicite ${ }^{65}$.

\footnotetext{
59. Voir aussi S. Besson, op.cit., 2016; S. Besson, «Concurrent responsibilities under the European Convention on human rights: The concurrence of human rights jurisdictions, duties and responsibilities", op. cit., 2018.

60. Cf. M. Den Heijer M., "Procedural aspects of shared responsibility in the European Court of Human Rights», Journal of International Dispute Settlement, vol. 4, nº 2, 2013, p. 361-383.

61. Cf. par exemple CourEDH, Ilaşcu et autres c. Moldova et Russie, arrêt du 8 juillet 2004, Rec. 2004VII, ECLI:CE:ECHR:2004:0708JUD004878799; CourEDH, Catan et autres c. République de Moldova et Russie, arrêt du 19 octobre 2012, Rec. 2012-V, ECLI:CE:ECHR:2012:1019JUD004337004; CourEDH, Rantsev c. Chypre et Russie, arrêt du 7 janvier 2010, Rec. 2010-I, ECLI:CE:ECHR:2010:0107JUD002596504; CourEDH, M.S.S.

62. Cf. par exemple CourEDH, Loizidou c. Turquie, arrêt du 18 décembre 1996, Rec. 1996-VI, ECLI:CE:ECHR:1996:1218JUD001531889; CourEDH, Sargsyan c. Azerbaïdjan, arrêt du 16 juin 2015, Rec. 2015, ECLI:CE:ECHR:2015:0616JUD004016706; CourEDH, Chiragov et autres c. Arménie, arrêt du 16 juin 2015, Rec. 2015, ECLI:CE:ECHR:2015:0616JUD001321605; CourEDH, Hassan c. RoyaumeUni, arrêt du 16 septembre 2014, Rec. 2014, ECLI:CE:ECHR:2014:0916JUD002975009; CourEDH, Jaloud c. Pays-Bas, arrêt du 20 novembre 2014, Rec. 2014, ECLI:CE:ECHR:2014:1120JUD004770808; CourEDH, El-Masri c. «l'Ex-République Yougoslave de Macédoine», arrêt du 13 décembre 2012, Rec. 2012-VI, ECLI:CE:ECHR:2012:1213JUD003963009; CourEDH, Al Nashiri c. Pologne, arrêt du 24 juillet 2014, ECLI:CE:ECHR:2014:0724JUD002876111; CourEDH, Roman Zakharov c. Russie, arrêt du 4 décembre 2015, Rec. 2015, ECLI:CE:ECHR:2015:1204JUD004714306.

63. Cf. par exemple CourEDH, Senator Lines GmbH c. Autriche, Belgique, Danemark, Finlande, France, Allemagne, Grèce, Irlande, Italie, Luxembourg, Pays-Bas, Portugal, Espagne, Suède et Royaume-Uni, décision du 10 mars 2004, Rec. 2004-IV, ECLI:CE:ECHR:2004:0310DEC005667200; CourEDH, Banković; CourEDH, Mohammed Hussein et autres c. Pays-Bas et Italie, décision du 2 avril 2013, ECLI:CE:ECHR:2013:0402DEC002772510.

64. Cf. par exemple CourEDH, M.S.S., \$ 341-348; CourEDH, Rantsev, $\$ 404-411$.

65. Cf. par exemple CourEDH, Ilascu, $\$ 484-490$.
} 
Les raisons de cette négligence sont multiples. Les traités internationaux de protection des droits de l'homme ont un champ d'application personnel et territorial limité puisqu'ils sont surtout régionaux. C'est notamment le cas de ceux qui disposent de tribunaux à juridiction obligatoire, comme la CourEDH. Tous les États responsables ne sont donc pas forcément membres des mêmes instruments ${ }^{66}$. C'est certainement le cas des OI qui n'ont d'obligations en droit international des droits de l'homme qu'en vertu de leurs règles internes, lorsqu'elles existent à cet égard ${ }^{67}$. Il demeure donc encore difficile à ce jour pour une victime de violation des droits de l'homme de rechercher plusieurs États/OI en responsabilité. Pour le cas où ce serait possible, encore faudrait-il que la requête remplisse les conditions de recevabilité pour chacun des États/OI responsables, ce qui peut être fastidieux. Il faudrait par exemple établir que chacun des États recherchés remplit la condition de juridiction au sens de l'art. 1 de la $\mathrm{CEDH}^{68}$.

\subsubsection{La critique du régime de la responsabilité solidaire internationale}

Le caractère restrictif du régime de responsabilité solidaire et son application limitée en droit international des droits de l'homme est source d'injustice.

C'est le cas en premier lieu pour les victimes individuelles des violations. Il existe un risque de réparation incomplète du préjudice subi en fonction de l'État / de l'OI recherché(e) en responsabilité. Chacun(e) répondra pour sa part de responsabilité seulement, et pour autant qu'on puisse faire reconnaître sa responsabilité. L'intérêt des victimes à être dédommagées devrait pourtant être considéré comme plus important que l'éventuel risque couru par responsables recherchés de ne pas pouvoir se voir ensuite remboursés par les autres responsables.

La situation est aussi source d'injustice pour les auteurs des violations. Il existe un risque d'enrichissement illégitime de l'État / de l'OI qui n'est pas recherché(e) en responsabilité au détriment de celui / de ceux qui le sont, et, à l'inverse, un risque pour l'État / l'OI recherché(e) d'encourir une

\footnotetext{
66. Cf. par exemple les USA dans CourEDH, Hassan ou CourEDH, El-Masri.

67. Cf. par exemple l'ONU dans CourEDH, Behrami; CourEDH, Al-Jedda c. Royaume-Uni, arrêt du 7 juillet 2011, Rec. 2011-IV, ECLI:CE:ECHR:2011:0707JUD002702108; CourEDH, Nada c. Suisse, arrêt du 12 septembre 2012, Rec. 2012-V, ECLI:CE:ECHR:2012:0912JUD001059308; ou l'UE dans CourEDH, Bosphorus Hava Yollari Turizm Ve Ticaret Anonim Sirketi c. Irlande, arrêt du 30 juin 2005, Rec. 2005-VI, ECLI:CE:ECHR:2005:0630JUD004503698.

68. Cf. par exemple CourEDH, Jaloud, $\$ 153$, qui n'examine pas à nouveau les conditions relatives à la juridiction du Royaume-Uni, ayant établi celle des Pays-Bas. Voir aussi M. Den Heijer, "Procedural aspects of shared responsibility in the European Court of Human Rights », op. cit., 2013; S. Besson, op. cit., 2016.
} 
responsabilité supérieure à sa responsabilité de fait, et ce parce qu'il est le seul pour lequel un effort d'attribution est réalisé, avec les risques de distribution injuste de responsabilité que cela implique. On remarque en effet en pratique que lorsqu'un seul responsable est recherché, la responsabilité des autres au même préjudice n'est pas prise en compte et qu'il y a une tendance à le faire répondre d'une part plus importante du préjudice que celle qu'il a effectivement causée ${ }^{69}$.

Ce risque devient d'autant plus préoccupant que les États agissent dans toujours davantage de cas de concert, et que les situations transnationales de violation du droit international augmentent de jour en jour. En outre, le nombre grandissant d'OI et de leur champ d'activités rend l'implication d'une ou de plusieurs OI et d'un ou de plusieurs États désormais routinière. La question de la répartition des responsabilités plurales est donc brûlante, ce qui implique d'examiner plus à fond la possibilité d'étendre le champ d'application de la responsabilité solidaire.

\section{L'extension et la consolidation de la responsabilité solidaire en droit international}

En réponse à cette difficulté, il s'agit de proposer, dans cette section, une interprétation visant à étendre l'applicabilité de la responsabilité solidaire dans le contexte des violations transnationales du droit international des droits de l'homme.

\subsection{L'extension du champ d'application de la responsabilité solidaire}

Les conditions d'application en droit international du principe de responsabilité solidaire pourraient tout d'abord être facilement étendues. Deux d'entre elles pourraient être retenues de manière alternative.

Premièrement, il s'agirait, comme actuellement, de faire dépendre la responsabilité solidaire de l'existence d'un même fait illicite (art. 47[1] ARSIWA et 48[1] DARIO). Les raisons sont celles déjà avancées précédemment, et notamment l'existence d'une causalité complexe lorsque le fait illicite est conjoint, d'une part, et la possibilité d'une coordination ex post entre responsables vu qu'ils ont pu se coordonner ex ante pour violer le droit international, d'autre part.

Deuxièmement, on pourrait ajouter une deuxième condition alternative : l'existence d'un cas de causalité cumulative - et ce, même en cas

69. Voir notamment dans la jurisprudence de la CourEDH, S. Besson, op. cit., 2016. 
de faits illicites distincts, que ces derniers soient d'ailleurs identiques ou non. Cette nouvelle condition serait justifiée par l'impossibilité de séparer les faits illicites distincts lorsqu'ils ont causé le préjudice de manière cumulative ${ }^{70}$. En bref, la causalité est parfois si complexe que les responsabilités ne peuvent pas être désagrégées et distribuées et que les autres critères de distribution, comme la cause adéquate ou le principe d'équivalence des causes, ne sont pas justes ou convaincants du point de vue de la victime de la violation. Tout au plus sont-ils intéressants pour répartir les recours internes ensuite, comme nous le verrons. Par extension, on peut aussi ajouter que lorsque plusieurs faits illicites distincts ont causé de manière cumulative un même préjudice, c'est que les responsables ont agi de manière coordonnée ou, en tout cas, auraient pu le faire. Cela justifie donc une solidarité dans les responsabilités ex post pour des raisons analogues à celles qui la justifient en cas de fait illicite conjoint.

On peut avancer trois justifications pour cette extension du champ d'application du principe de responsabilité solidaire en cas de responsabilités partagées par plusieurs États et/ou OI.

Premièrement, la nature des sujets lésés, à savoir, des individus. Contrairement à ce qui prévaut en matière de responsabilité internationale en général, les victimes de violations du droit international des droits de l'homme sont des particuliers, et non pas des États ou des OI. Ceci rend la question de la réparation complète du tort qui leur a été causé et de la justice de la distribution des réparations encore plus urgente. Une fois que l'égalité des sujets responsables et lésés, et surtout la nature horizontale de leur rapport, sont remplacées par le déséquilibre entre eux et la verticalité du rapport de violation institutionnelle des droits de l'homme et de la responsabilité induite, la solidarité entre États et/ou OI responsables envers les victimes individuelles paraît évidente.

Deuxièmement, l'existence d'une obligation positive générale d'institutionnalisation de la part des États, et donc déjà d'une véritable solidarité de leurs obligations primaires. En droit international des droits de l'homme, il est en effet possible de développer l'argument selon lequel, lorsque plusieurs États exercent une juridiction concurrente sur les mêmes personnes (soit un contrôle effectif, normatif et régulier ${ }^{71}$ ), ils encourent non seulement des obligations concurrentes relatives aux droits de l'homme de ces personnes, mais aussi une obligation positive générale de coordonner

70. Cf. aussi P.d'Argent, op. cit., p. 241-244. Voir aussi J.E. Noyes et B.D. Smith, op. cit., p. 262-266. 71. Cf. S. Besson, «The extraterritoriality of the European Convention on Human Rights: Why Human Rights depend on jurisdiction and what jurisdiction amounts to ", Leiden Journal of International Law, vol. 25, $\mathrm{n}^{\circ} 4,2012$, p. 857-884, particulièrement p. 860-861. 
leurs obligations respectives ${ }^{72}$. Chaque État a en effet l'obligation positive de protéger les personnes sous sa juridiction au mieux et cela inclut la prise en compte des menaces que font peser sur leurs droits d'autres États ayant juridiction concurrente sur elles ${ }^{73}$. Cela donne donc lieu à une obligation pour chaque État impliqué de coordonner l'allocation de ses obligations internes, si nécessaire par le biais de la création d'institutions communes. C'est particulièrement le cas lorsque les États ont agi dans un cadre institutionnel distinct, comme celui d'une OI (par exemple UE ou ONU). Dans de tels cas, les institutions nécessaires à la coordination de leurs obligations concurrentes sont en place et leur permettent de coordonner leur allocation. S'il existe une obligation positive des États de coordonner l'allocation de leurs obligations ex ante et leur mise en œuvre, les responsabilités pour violation de ces obligations doivent aussi pouvoir être coordonnées ex post et être dues de manière solidaire. En d'autres termes, la solidarité des responsabilités n'est alors que le reflet de la solidarité des obligations primaires des États.

Il ne faut pas confondre ces obligations positives de coordonner des obligations de droits de l'homme concurrentes entre États avec les obligations positives de chacun de ces États de protéger les titulaires de ces droits de l'homme contre d'autres acteurs comme d'autres États ou contre les OI. Dans ces cas-là, il n'y a même pas d'attribution de comportement ou de responsabilité, puisqu'un seul État répond de son propre fait illicite, à savoir, de ne pas avoir empêché les autres d'agir ${ }^{74}$. On peut néanmoins voir dans ces obligations positives un moyen de réduire la pluralité des responsabilités et de les concentrer en une seule, ce qui peut constituer une méthode pour les tribunaux internationaux de droits de l'homme d'éluder la question de la responsabilité partagée, et donc toute la question de la répartition des responsabilités ${ }^{75}$.

Troisièmement, l'existence de tribunaux ou organes internationaux de mise en cuvre des droits de l'homme, et dont la juridiction est obligatoire. Contrairement à ce qui prévaut en matière de responsabilité internationale en général, les responsabilités plurales pour violation des droits de l'homme peuvent être constatées par des tribunaux existants et compétents, d'une

\footnotetext{
72. Voir pour un argument complet, S. Besson, op. cit., 2016; S. Besson, «Concurrent responsibilities under the European Convention on human rights: The concurrence of human rights jurisdictions, duties and responsibilities", op. cit., 2018. Voir aussi S. Besson, "The allocation of anti-poverty rights duties - Our rights, but whose duties? », in K. Nadakavukaren Schefer (dir.), Poverty and the International Economic Legal System. Duties to the World's Poor, Cambridge, Cambridge University Press, 2013, p. 408-431.

73. Cf. par exemple CourEDH, Catan, $\$ 145$; CourEDH, Sargsyan, $\$ 131$.

74. Cf. par exemple CourEDH, Al-Nashiri, $\$ 517$; CourEDH, Soering, $\S 86-88$; CourEDH, Ilaşcu, $\$ 392-393$.

75. Cf. par exemple S. Besson, op. cit., 2016; M. Den Heijer, «Procedural aspects of shared responsibility in the European Court of Human Rights», op.cit., 2013.
} 
part, et des tribunaux dont la juridiction est obligatoire, d'autre part. Ceci permet donc de repousser la critique habituelle à l'égard du recours à la responsabilité solidaire, qui invoque l'absence de tribunaux aptes à organiser les recours internes ex post entre responsables.

\subsection{La mise en œuvre de la responsabilité solidaire étendue}

Une fois l'applicabilité de la responsabilité solidaire justifiée, il convient encore de se préoccuper de ses conséquences internes entre États et/ou OI responsables. L'un des reproches qui est souvent fait à son application en droit de la responsabilité internationale est en effet le caractère encore incertain de son régime ${ }^{76}$.

Il s'agit, tout d'abord, d'organiser l'ordre des recours internes entre États/OI solidairement responsables pour permettre à celui qui a réparé l'entier du préjudice de récupérer la part des autres. Les articles 47 ARSIWA / 48 DARIO mentionnent ces recours, mais n'en règlent ni l'ordre ni les critères. Ces questions n'ont en outre été qu'à peine effleurées dans la jurisprudence. Il semble découler de cette dernière, en effet, qu'il ne doit pas y avoir de cumul d'actions contre chaque État/OI responsable, et que les recours internes doivent être organisés ${ }^{77}$. En pratique, ces recours sont pourtant toujours organisés ex gratia $^{78}$ ou selon des accords ad hoc entre les États responsables. Certains régimes spéciaux les organisent, cependant. En l'absence d'accord spécifique des parties, ils prévoient notamment leur organisation par référence à la faute des États et/ou OI responsables. C'est ce que font les articles IV(2) et V(2) de la Convention de 1972 sur la responsabilité internationale pour les dommages causés par des objets spatiaux ${ }^{79}$.

Par défaut, et en l'absence d'accord des parties ou de régime spécial, on pourrait proposer les critères de base suivants pour l'allocation des recours internes. Ces critères se basent sur les critères de répartition des responsabilités elles-mêmes lorsqu'elles sont divises ${ }^{80}$. Ils peuvent être utilisés conjointement ou, sinon, de manière subsidiaire les uns aux autres, notamment dans l'ordre d'exposition.

Premièrement, la causalité entre le fait illicite et le préjudice. En cas de causalité exclusive ou parallèle, on peut prévoir des recours pour l'entier de la responsabilité dans la contribution au préjudice causé. Ce genre de causalité

\footnotetext{
76. Cf. P. d'Argent, op. cit., p. 244-249; Crawford, p. 355 sq.

77. Cf. CIJ, Détroit de Corfou, \$22-23; CPJI, Affaire relative à l'Usine de Chorzow (Demande en indemnité), arrêt du 13 septembre 1928, Séries A nº 17, $\$ 45-49$.

78. Cf. CIJ, Phosphates de Nauru, \$258-262.

79. Cf. S. Besson, op. cit., 2007, p. 18-19.

80. Cf. S. Besson, op. cit., 2007, p. 24-25; P. d'Argent, op. cit., p. 247-249.
} 
sera plutôt rare cependant dans des circonstances de fait illicite conjoint, et donc en cas de responsabilité solidaire. En cas de complémentarité des causes, par contre, on pourra prévoir des recours proportionnels à la quotepart du préjudice causé. À nouveau, toutefois, ce genre de causalité sera rare dans des circonstances de fait illicite conjoint, et donc de responsabilité solidaire. En cas de causalité cumulative, la question est plus difficile. Il est toujours possible toutefois de recourir à la sélection d'une cause "adéquate» reconstruite (par exemple la plus récente) ou à l'«équivalence» des causes, et donc à l'égalité des rapports internes.

Un deuxième critère pourrait être le type et/ou la force normative des obligations violées. En droit privé interne, en effet, on distingue entre la solidarité parfaite et imparfaite selon les chefs de responsabilité, selon qu'ils sont par exemple de nature similaire ou non ${ }^{81}$. Cette caractérisation influence ensuite le rôle que va jouer la faute ou d'autres éléments dans la répartition des recours internes. Un troisième et un quatrième critères pourraient précisément être ceux de la gravité des violations de ces obligations ou encore la faute. Aucun de ces trois derniers critères cependant ne se retrouve dans la pratique de la responsabilité internationale. Certains, comme la faute, sont même étrangers à sa structure, comme nous l'avons vu précédemment. Certains régimes spéciaux, toutefois, comme la Convention de 1972 sur la responsabilité internationale pour les dommages causés par des objets spatiaux, prévoient la faute comme élément d'appréciation des montants dus au titre de recours internes.

Enfin, parmi les critères complémentaires, on pourrait mentionner les fondements d'attribution de comportement et surtout de responsabilité euxmêmes, lorsque le concours de responsabilités est le fruit d'une attribution conjointe de comportement ou d'une attribution de responsabilité. Ces fondements d'attribution pourraient en effet fonctionner comme une sorte de causalité de deuxième ordre, ou de «causalité dans la causalité ». Ainsi, par analogie avec l'art. 39 ARSIWA / art. 39 DARIO quant à la contribution du lésé à son préjudice, la contribution au préjudice par les autres États/ OI responsables devrait pouvoir être prise en compte dans l'examen des responsabilités respectives de chacun. Ces contributions respectives pourraient corriger la répartition causale des responsabilités internes, voire se substituer à celle-ci. Ainsi, en cas d'attribution de responsabilité du fait de la contrainte ou des directives, c'est le degré de contrôle d'un responsable sur l'autre qui pourrait être utilisé, sachant que les responsabilités des uns et des autres sont proportionnelles au contrôle exercé ou, à l'inverse, au contrôle subi ${ }^{82}$. En cas d'attribution de responsabilité du fait de l'assistance ou de la complicité, c'est le degré d'assistance d'un responsable sur l'autre

81. Cf. S. Besson, op. cit., 2007, p. 14 et 25.

82. Cf. J.D. Fry, op. cit., p. 127-133. 
qui importerait, et donc du fait illicite d'aider sur le fait illicite distinct de celui qui est aidés3.

Il demeure bien sûr de nombreuses autres questions procédurales à résoudre si le régime de la responsabilité solidaire est étendu et renforcé, comme je l'ai proposé. Les articles 47 ARSIWA / 48 DARIO les mentionnent, mais ne les règlent pas. On évoquera notamment le régime légal relatif aux recours internes (par exemple: ordre, compensation, prescription, etc.); la question des procédures judiciaires adéquates pour les recours internes et la répétition de l'indu en cas d'enrichissement illégitime ${ }^{84}$; ou encore les règles relatives au cumul d'actions et à la libération des autres co-responsables par le paiement de l'un d'entre eux.

Il y a différents moyens de répondre à ces questions procédurales dans l'immédiat et en l'absence d'accord particulier entre responsables, de régime spécial ou de règles internes aux OI. Il faut mentionner notamment les principes généraux de droit et le recours au droit national comparé de la responsabilité pour les identifier ${ }^{85}$. Les conditions de reconnaissance d'un principe général de droit en droit international sont la généralité du principe en droit national et la transposabilité au droit international. On a bien sûr critiqué les analogies rapides avec le droit national de la responsabilité civile et mis en cause le degré de généralité de ces principes, mais aussi leur adéquation au contexte des relations internationales ${ }^{86}$. Il semble toutefois que, pour autant qu'il soit possible d'établir un degré de cohérence suffisant entre traditions nationales de la responsabilité civile et généralité de certains principes minimaux, ce problème puisse être surmonté. Quant au problème des analogies avec le droit privé de la responsabilité et de sa transposition à des acteurs publics comme les États et les OI, il subsiste, bien sûr. Il s'agit cependant là d'une question qui remonte aux choix opérés dans la pratique internationale et lors de la codification des ARSIWA/DARIO en général.

\section{La responsabilité solidaire des États membres pour leur OI : difficultés et possibilités}

On peut être tenté de considérer que, puisque les OI responsables d'un fait internationalement illicite sont constituées par leurs États membres en vue de remplir certaines fonctions que ceux-là leur donnent, ces États devraient automatiquement porter la responsabilité des faits illicites de

\footnotetext{
83. Cf. H. Aust, op. cit., p. 294-295.

84. Cf. C. Binder et C. Schreuer, «Unjust Enrichment», in R. Wolfrum (dir.), Max Planck Encyclopedia of International Law, vol. X, Oxford, Oxford University Press, 2007, p. 588-597.

85. Cf. par exemple l'opinion individuelle du Juge Simma dans CIJ, Plates-formes pétrolières, $\$ 66$ et 74. Cf. également S. Besson, op. cit., 2007, p. 22-24; J.E. Noyes et B.D. Smith, op. cit., p. 252-253. 86. Cf. P. d'Argent, op. cit., p. 244-246; Crawford, p. 328-332.
} 
leurs OI, conjointement ou même solidairement avec ces dernières, et ce simplement en vertu de leur qualité d'État membre.

Il convient de distinguer cette question des responsabilités éventuelles des États en cas de responsabilité exclusive pour fait illicite d'une OI dont ils sont membres de celle de la responsabilité conjointe, voire même solidaire de plusieurs États et OI, que ce soit par attribution de comportement ou de responsabilité (art. 58-62 DARIO), notamment en cas de contournement des obligations des États (art. 61 DARIO). Il ne s'agit pas, en effet, dans cette section, de considérer les cas dans lesquels les faits illicites d'une OI sont aussi attribués, principalement ou en plus, aux États membres de cette OI, soit par attribution de comportement conjointe soit par attribution de responsabilité, comme nous l'avons vu précédemment, mais des cas dans lesquels seule l'OI est responsable du fait illicite du point de vue du droit de la responsabilité internationale. Il faut d'ailleurs souligner que ces cas sont rares tant il est inhabituel qu'une OI soit seule responsable sous DARIO et ce, en raison de la multitude des fondements d'attribution de comportement et de responsabilité d'une OI à un ou plusieurs États en même temps. On peut même dire que c'est le propre des DARIO que d'avoir organisé les attributions de responsabilité de l'OI aux États membres de manière à prendre en compte le contrôle qu'exercent les États sur les OI.

\subsection{La difficulté du voile institutionnel}

En principe, il n'y a pas de responsabilités mutuelles ou cumulatives, même subsidiaires, entre un ou plusieurs États membres d'une OI et cette OI du simple fait de l'appartenance des premiers à la seconde. La raison réside dans ce que l'on appelle le «voile corporatif» ou « institutionnel ${ }^{87}$ ». Ce voile protège l'autonomie et la personnalité juridique distincte de l'OI de celle de ses États membres ${ }^{88}$, comme il protège, en droit des sociétés national, l'autonomie et la personnalité distincte des sociétés de celle de leurs membres individuels.

C'est ce que confirme l'article 62 DARIO a contrario: en l'absence de fondement d'attribution de responsabilité des articles $58 \mathrm{sq}$. DARIO, un État ne répond du fait illicite d'une OI que par sa reconnaissance de cette responsabilité. Il faut donc soit un fondement d'attribution de comportement de l'un à l'autre soit un fondement d'attribution de

\footnotetext{
87. Cf. par exemple C. Broelmann, The Institutional Veil in Public International Law: International Organisations and the Law of Treaties, Oxford, Hart, 2007; C. Ryngaert. et H. Buchanan, «Member State Responsibility for the Acts of International Organizations », Utrecht Law Review, vol. 7, n 1, 2011, p. 131-146; Crawford, op.cit., p. 343-344.

88. L'étymologie du terme personnalité est d'ailleurs le masque, le rôle ou le voile (persona en latin).
} 
responsabilité de l'un à l'autre pour qu'un ou plusieurs États répondent du fait illicite d'une OI.

Même en cas d'organes partagés, le contrôle doit être conjoint sur ces organes pour que l'attribution de comportement aux deux soit retenue (art. 7 $\mathrm{DARIO}^{89}$ ). Le propre des rapports d'une OI et des États membres, en effet, est que ces derniers (plus précisément, les organes de ces derniers) fonctionnent parfois comme les organes de l'OI, mais pas nécessairement. À l'inverse, lorsque les organes de l'État fonctionnent en tant qu'organes de l'OI, ce n'est en principe pas en même temps en tant qu'organes de l'État. Quant à l'attribution de responsabilité d'une OI à un ou plusieurs États, elle peut se fonder sur l'assistance, les directives, la contrainte ou encore le contrôle (art. 58-60 DARIO). Le fondement d'attribution de responsabilité qui se rapproche le plus d'une attribution mutuelle de responsabilité entre une OI et ses États membres est le contournement d'obligations (art. 61 DARIO). Cependant, même dans ce cas, il faut encore établir l'intention des États membres de contourner leurs obligations, par exemple en déléguant certaines de leurs compétences à l'OI.

Pourtant, nos intuitions morales résistent aux conséquences du voile institutionnel pour la responsabilité des OI et à la protection indirecte des États membres de ces OI, comme elles résistent d'ailleurs au voile corporatif qui protège l'irresponsabilité des membres individuels ou actionnaires d'une société lorsque cette dernière est responsable. Les raisons de cette résistance sont en large partie les mêmes. Elles sont avant tout financières: il se peut en effet qu'en l'absence de moyens de se retourner contre les États membres, l'OI ne puisse pas honorer ses responsabilités, comme ce peut être le cas d'une société commerciale en faillite et comme la réalité internationale l'a déjà démontré ${ }^{90}$.

En fait, face à une OI, l'intuition morale de l'injustice du voile institutionnel est d'autant plus forte que, d'une part, les OI n'ont que peu d'obligations primaires de droit international, et notamment pas d'obligations de droits de l'homme, et que, d'autre part, les OI ne sont pas ou que très peu soumises à la juridiction de tribunaux nationaux (du fait, pour ces derniers, des immunités juridictionnelles des OI) ou internationaux (mis à part à leurs propres tribunaux internes, quand elles en ont, et pour des

89. Cf. par exemple CourEDH, Al-Jedda, $\$ 76 s q$.

90. Cf. la décision britannique House of Lords, International Tin Council: J.H. Rayner (Mincing Lane) Ltd v. Department of Trade and Industry and others and related appeals; Maclaine Watson \& Co Ltd v. Department of Trade and Industry, and Maclaine Watson \& Co. Ltd v. International Tin Council, décision du 26 octobre 1989, International Law Reports, vol. 81, 1990, p. 670-725. 
questions administratives ou privées principalement ${ }^{91}$ ). Il est donc très difficile de les tenir responsables en pratique, même si les conditions de DARIO sont remplies. Cette «irresponsabilité » généralisée des OI envers les tiers est donc soit le fruit de l'absence d'obligations à violer, dont elles devraient alors répondre, et par conséquent de responsabilité objective, soit le résultat d'un manque de moyens procéduraux, et notamment judiciaires, de faire reconnaître cette responsabilité (subjective) en dépit de l'existence de la responsabilité objective. Sans responsabilité automatique ou mutuelle de leurs États membres, l'irresponsabilité serait donc complète ${ }^{92}$. C'est cela qui distingue, par conséquent, la relation États/OI de la relation actionnaires/société : les obligations et responsabilités des premiers sont différentes en nature et régime, alors qu'elles sont similaires et interchangeables pour les deuxièmes. Si l'on peut comprendre le remplacement d'un responsable par l'autre dans le deuxième cas, il est plus difficile à justifier dans le premier. Dans ce cas, en effet, le voile institutionnel sert d'échappatoire à toute responsabilité.

À cet égard, il est intéressant de remarquer que nos intuitions vont à l'encontre du voile institutionnel lorsqu'il s'agit des responsabilités des OI et de l'irresponsabilité induite des États membres, alors qu'elles vont dans le sens contraire lorsqu'il s'agit des responsabilités des États et de l'irresponsabilité des particuliers qui les constituent. Après tout, la responsabilité des États a des conséquences automatiques pour celle des individus qui en sont membres dans la mesure où ce sont ces derniers qui financent les réparations en cas de responsabilités de guerre, par exemple, et alors même qu'il ne s'agit pas des individus qui ont pris les décisions à l'origine de la responsabilité de ces États (leur responsabilité pénale individuelle est distincte en droit international). C'est ce qu'on appelle parfois le «défi individualiste» (individualist challenge) au principe de la responsabilité internationale des États ${ }^{93}$.

Pourtant, on rencontre peu de contestation de la justice de cette absence de voile institutionnel de l'État et le défi individualiste n'est pas souvent pris au sérieux. Une première explication de l'absence de voile institutionnel en faveur des citoyens d'un État est le lien politique, et notamment démocratique, qui lie la coordination de l'action politique à celle de la responsabilité collective. Ce lien fait que tous les membres individuels de l'État acceptent de

91. Cf. par exemple l'article 29 de la Convention sur les privilèges et immunités des Nations unies, New York, 13 février 1946, UNTC 4. Voir J. Klabbers, "The transformation of international organizations law», European Journal of International Law, vol. 26, $\mathrm{n}^{\circ} 1,2015$, p. 9-82, en particulier p. 65 sq.; J. Alvarez, "The United Nations in the time of cholera», https://www.asil.org/blogs/ united-nations-time-cholera (consulté le 23 août 2016).

92. Pour cette critique, voir J. Klabbers p. 68-71 et 72-74.

93. Cf. L. Murphy, «International responsibility», in S. Besson et J. Tasioulas (dir.), The Philosophy of International Law, Oxford, Oxford University Press, 2010, p.299-315. Contra: J. Crawford et J. Watkins, «International responsibility», in: ibid., p. 283-298. 
porter le poids de la responsabilité de l'État ou, du moins, devraient le faire. On peut même parler de «solidarité démocratique» à cet égard. D'autres explications sont que c'est le propre de l'action collective, et donc du projet étatique, que de créer un agent collectif autonome, ou encore que la durabilité dans le temps de l'action collective requiert l'existence d'un agent autonome. Finalement, comme mentionné précédemment lorsqu'il était question de la dis-analogie entre le voile corporatif au profit des actionnaires de sociétés privées et le voile institutionnel au profit des États membres d'OI, les obligations et responsabilités des citoyens individuels et de leurs États ne sont pas les mêmes, ce qui ne les rend pas interchangeables.

Dès lors, pourquoi tant d'insistance sur le voile institutionnel des OI lorsque ce dernier protège l'irresponsabilité des États? Curieusement, en effet, les éléments relatifs au caractère public de l'entité étatique, absents dans le cas d'une société privée, sont bien présents dans la relation États/ OI. C'est le cas notamment de l'action collective et de la durabilité de cette action. Quant à la question du lien politique, voire démocratique, il est plus difficile à établir directement entre les OI et les États, voire même entre les OI et les individus citoyens, en l'absence de communautés politiques internationales au-delà des États (même s'il y a des variations entre les OI comme, par l'exemple, l'ONU et l'UE). En revanche, il est clairement à l'œuvre entre les États membres et leurs citoyens, et ce même lorsque les premiers agissent comme membres d'une OI. Leurs responsabilités (envers leurs membres individuels) ne devraient pas pouvoir être si facilement écartées en cas de constitution d'une OI, par conséquent, et encore moins celles des individus qui en sont membres, par extension de la responsabilité collective individuelle pour les États.

Parmi les explications de la différence de traitement à cet égard de la relation de membre États/OI et individus/État, on avance habituellement la tension qui existe entre la personnalité et l'indépendance internationale des OI et celle des États. Lever le voile institutionnel reviendrait à leur faire perdre cette indépendance qui se trouve en concurrence avec celle de leurs États membres. Au vu des liens intenses entre responsabilité et personnalité en droit ${ }^{94}$, cette relation se répercute donc en matière de responsabilité des OI. En somme, ce serait pour protéger les OI des États qu'il leur faudrait un voile institutionnel en matière de responsabilité.

Cette explication ne constitue cependant pas une justification suffisante ${ }^{95}$. D’une part, il convient de rappeler la différence de nature et de régime entre les obligations et responsabilités des OI et des États mentionnées plus haut

94. Cf. CIJ, Immunité de juridiction d'un rapporteur spécial de la Commission des droits de l’homme, $\$ 66$. 95. Pour un argument complet, voir S. Besson, «Individual and State liability for an international organization's responsibility. The challenge of fairness unveiled ", Italian Journal of Legal Philosophy, VI/1, 2017, p. 51-78. 
lorsqu'a été évoquée la dis-analogie avec le voile corporatif. Comme nous l'avons vu, les responsabilités des OI et des États ne sont pas interchangeables, et ce notamment pour des raisons politiques et démocratiques aussi. Alors que les premières ne répondent pas (encore) démocratiquement de leurs actes, les seconds le font. Le voile institutionnel, protecteur d'autonomie des OI, ne serait dès lors guère plus qu'un voile d'irresponsabilité pour les États. D'autre part, l'autonomie des OI implique, pour qu'elles soient dignes de protection en droit international, des obligations et des responsabilités comparables à celles des États, ou du moins complètes, même si elles en sont distinctes. Tant que ce n'est pas le cas, défendre le caractère indépendant de leur responsabilité ne fait que protéger l'inaction des États et les disculper de leurs responsabilités.

\subsection{Quelques trouées solidaires dans le voile institutionnel}

Face à la ténacité de l'intuition morale relative à la responsabilité des États membres d'une OI pour les faits illicites de cette dernière, il convient de mentionner quelques pistes juridiques à explorer, notamment en lien avec mon argument sur la responsabilité solidaire des États/OI. Il s'agit d'identifier les moyens de lever partiellement le voile institutionnel, par conséquent, mais sans menacer l'autonomie des OI et sans remettre en cause leur responsabilité propre, et donc sans tenir les États membres d'une OI automatiquement et mutuellement responsables pour son fait sans motif d'attribution. Au contraire, ce qui les rendrait responsables, selon cette proposition, serait leur fait en tant qu'État membre d'une OI et leur solidarité avec ses actions, mais au-delà des fondements d'attribution de comportement et de responsabilité existants dans les DARIO.

La première possibilité à exploiter davantage est celle des obligations de solidarité financière des États membres de l'OI du fait de l'article 40(2) DARIO. Cette disposition fait écho à la tragédie de la faillite du Conseil international de l'étain en 1985, qui a laissé beaucoup de créances impayées ${ }^{96}$. Si une OI dispose de l'indépendance juridique suffisante pour être responsable (ou, à l'inverse, de la responsabilité suffisante pour être indépendante juridiquement), tel n'est pas forcément le cas de son indépendance financière, puisqu'elle dépend largement de ses États membres sur ce plan.

En réponse à cette préoccupation, l'article 40(1) DARIO exige que les OI s'organisent de manière à ce que leurs États membres leur assurent les moyens financiers de leurs responsabilités: il crée donc une obligation primaire de

96. House of Lords, International Tin Council. Voir aussi C. Ahlborn «The rules of international organizations and the law of international responsibility", International Organizations Law Review, vol. 8, nº 2, 2011, p. 397-482; J. Klabbers, op. cit., p. 63-64. 
financement pour les OI qui doivent adopter du droit interne pour la réaliser. L'article 40(2) DARIO crée une autre obligation primaire de financement de l'OI, mais pour les États membres cette fois-ci, et cette obligation est une obligation de droit international de la responsabilité directement, même si sa réalisation est encore une fois soumise à l'adoption de droit interne par l'OI. On peut considérer cette obligation comme une obligation collective et, du fait de la coordination institutionnelle en place, considérer que si elle est violée par un même fait illicite des États membres, cette responsabilité des États membres devra être solidaire ${ }^{97}$.

Bien sûr, cette responsabilité solidaire des États membres est une responsabilité des États membres de ne pas avoir fait en sorte que le droit interne de leur OI se dote d'obligations financières, et non pas une responsabilité relative au fait illicite de l'OI lui-même. Elle ne porte donc pas sur la dette elle-même de l'OI. En fait, on peut même avancer l'argument selon lequel l'autonomie juridique de l'OI dépend de son existence financière et donc de l'obligation collective, respectivement de la responsabilité solidaire de ses États membres de lui fournir ce soutien financier. La responsabilité des États membres et l'autonomie de l'OI ne sont donc pas en tension ici, bien au contraire.

La seconde possibilité est de considérer que, lorsque leur coopération est institutionnalisée sous la forme d'une OI, les États membres de cette OI répondent solidairement des actes de cette dernière s'ils n'ont pas veillé à ce que le droit de l'OI ne les amène pas à violer leurs obligations primaires de droit international (par exemple de droit international des droits de l'homme). Cela vaudrait même hors des hypothèses des articles 58 à 62 DARIO, et notamment hors du cas du contournement d'obligations et de l'intention des États de les contourner ${ }^{98}$. C'est en ce sens qu'on peut comprendre l'obligation de diligence des États membres d'une OI dans la $\mathrm{CEDH}$ et leur responsabilité pour ne pas avoir veillé à ce que l'OI assure une protection équivalente des droits à celle de la $\mathrm{CEDH}^{99}$.

Â nouveau, cette responsabilité ne serait pas une responsabilité pour le fait illicite de l'OI, mais pour le propre fait des États membres: ne pas avoir veillé à ce que leurs propres obligations primaires de diligence ne soient pas violées par l'activité de l'OI et ce, même sans intention de contournement

\footnotetext{
97. Cet argument a certes été expressément rejeté par Lord Oliver of Aylmerton dans House of Lords, International Tin Council, $\$ 706$, mais la décision est antérieure aux ARSIWA.

98. Pour une proposition légèrement différente, voir aussi J. d'Aspremont, "Abuse of the legal personality of international organizations and the responsibility of member states", International Organizations Law Review, vol. 4, nº 1, 2007, p. 91-119.

99. Cf. ComEDH, M \& Co. c. République fédérale d'Allemagne, décision du 9 février 1990, D.R. n 64, p. 138-146; CourEDH, Bosphorus, $\$ 152-155$; CourEDH, Al-Dulimi et Montana Management Inc. c. Suisse, arrêt du 26 novembre 2013, ECLI:CE:ECHR:2013:1126JUD000580908; CourEDH, Al-Dulimi et Montana Management Inc. c. Suisse, arrêt du 21 juin 2016, opinion concordante de la juge Keller, ECLI:CE:ECHR:2016:0621JUD000580908, $\$ 10$ J Klabbers $s q$.
} 
de leurs obligations par le biais du droit et de décisions de l'OI au sens de l'article 61 DARIO. La violation de cette obligation collective des États devrait être solidaire. En ce sens, la procédure intentée par certaines victimes contre les États membres de l'UE devant la CourEDH dans l'affaire Senator Lines était la bonne ${ }^{100}$, même si elle n'a pas eu gain de cause à l'époque et a fini par donner naissance à la présomption d'équivalence de l'arrêt Bosphorus ${ }^{101}$.

Pour ne pas menacer l'autonomie de l'OI, cependant, on pourrait considérer, comme à l'article 62(2) DARIO, que, dans de tels cas, la responsabilité solidaire des États membres de l'OI devrait être subsidiaire à celle de l'OI qui porterait la responsabilité primaire (sur la base de l'article 62 [2] DARIO a fortiori). Ainsi, lorsque l'OI a des obligations primaires et peut répondre de leur violation, c'est elle qui est responsable (sauf faits illicites concurrents des États membres, bien sûr). Dans les autres cas (la plupart, actuellement, en matière de droits de l'homme puisqu'il n'y a pas d'obligations des OI), c'est aux États membres de répondre subsidiairement et solidairement de son fait illicite.

\section{Conclusions : vers un nouveau droit de la responsabilité des États et des OI?}

Même révisé selon les propositions faites ici en vue d'une extension du champ d'application de la responsabilité solidaire entre États et/ou OI, le régime de responsabilité internationale des États et des OI attend une réforme en profondeur.

À la lumière des exemples récents, c'est surtout le régime de responsabilité internationale des OI qui doit être mis en cause et réformé. On pensera à l'absence de responsabilité de l'ONU pour l'épidémie de choléra en Haïti ou du FMI et de l'UE pour leur contribution à l'appauvrissement de la population grecque. On observe en effet tout d'abord un manque d'obligations primaires des OI envers d'autres États que leurs membres et envers les individus. Par exemple, ni l'ONU ni le FMI n'ont d'obligations de droits de l'homme en droit international. On remarque aussi, deuxièmement, un manque de remèdes judiciaires nationaux, du fait des immunités, et internationaux. Par exemple, ni l'ONU ni le FMI ne peuvent être soumis à des tribunaux nationaux en vertu de leurs immunités juridictionnelles et aucun d'entre eux n'est soumis à la juridiction de la CIJ ou d'un autre organe judiciaire international excepté quelques tribunaux civils ou administratifs qu'ils mettent en place exceptionnellement pour des cas limités.

100. CourEDH, Senator Lines.

101. CourEDH, Bosphorus. 
Par conséquent, les responsabilités des OI sont rarement reconnues en pratique et il y a un "vide de responsabilité ». Ce vide rend les efforts proposés dans cette contribution en matière de responsabilité solidaire des États et/ou des OI, d'une part, et de responsabilité solidaire additionnelle des États membres des OI, d'autre part, relativement vains, dans la mesure où la responsabilité internationale des OI n'existe souvent tout simplement pas. Si l'on souhaite amener des changements en profondeur, il convient donc de s'atteler aux problèmes inhérents au régime de la responsabilité internationale des OI lui-même.

À ce jour, différentes propositions ont été faites pour remplir le vide juridique causé par le régime de responsabilité internationale actuel des OI et l'irresponsabilité des OI qui s'ensuit la plupart du temps. Certains prônent, pour échapper à l'absence d'obligations primaires des OI et donc de responsabilité secondaire de ces dernières, le développement de formes alternatives de responsabilité des OI sans obligations primaires dont il faudrait établir la violation (par ex. qua accountability) ou encore de «responsabilités» en tant que sources d'obligations primaires (par exemple responsibility to protect). D'autres envisagent d'exploiter davantage les canaux de la réputation (shaming and blaming) et du discours de responsabilisation hors du droit de la responsabilité102. D'autres, enfin, poussent vers un retour à l'éthique et aux obligations et responsabilités éthiques des $\mathrm{OI}^{103}$.

Même si toutes ces propositions sont intéressantes et méritent d'être poursuivies, il y a cependant un danger à procéder uniquement de la sorte: c'est celui de jeter le bébé avec l'eau du bain. Tout d'abord, ces propositions se font au mépris de la question des obligations primaires des États et des OI (et donc des sources du droit international et de sa légitimité); au mépris, ensuite, du régime de droit de la responsabilité internationale existant; au mépris, enfin, du rôle bénéfique des OI dans la coordination des États pour la protection du droit international (y compris de la paix et des droits de l'homme). Mieux vaut dès lors essayer d'interpréter et de réformer le régime actuel de responsabilité des États et/ou des OI de l'intérieur pour en renforcer la légitimité, plutôt que de le contourner entièrement. Le droit et sa pratique comprennent leurs propres critiques et justifications internes qu'il s'agit d'identifier et de mettre au profit de ce travail de réforme. Il faut donc éviter l'erreur très répandue qui consiste à aborder un régime juridique comme un ensemble moralement inerte et sans justifications morales propres.

Parmi les propositions de réforme à examiner et développer, on mentionnera les pistes suivantes.

102. Cf. par exemple K. Daugirdas « Reputation and the responsibility of international organizations », European Journal of International Law, vol. 25, n 4, 2014, p. 991-1018.

103. Cf. par exemple J. Klabbers, op.cit., p. 75 sq. 
Premièrement, il faudrait commencer par mettre en cause la théorie fonctionnaliste qui a longtemps prédominé en droit international des OI, qu'il s'agisse de leur personnalité, de leurs immunités ou de leur responsabilité. En bref, les droits et obligations des OI sont conçus de manière à permettre à l'OI de remplir ses fonctions, fonctions qui sont présumées bonnes et dont la réalisation est présumée ne pas pouvoir entraîner la violation d'autres obligations (pour autant qu'elles existent, d'ailleurs, puisque l'OI a pour obligation principale de remplir ses fonctions ${ }^{104}$ ). La responsabilité des OI, lorsqu'elle existe, se limite donc à celle qui découle de la violation de leurs fonctions, et non pas des conséquences d'une telle violation. Elle est en outre principalement tournée vers l'intérieur (et ses États membres), et non pas l'extérieur et les sujets tiers (États tiers, autres OI ou individus).

Le fonctionnalisme n'a de justification, cependant, que si les États sont responsables des fonctions qu'ils attribuent aux OI et surtout des conséquences de la réalisation (même adéquate) de ces fonctions sur les tiers, et notamment les individus, puisque ce sont leurs intérêts (et donc ceux de leurs citoyens) que ses fonctions doivent servir. À partir du moment où ce n'est plus le cas, l'idée de fonctions d'une OI est creuse et ses fonctions ne sont qu'une coquille vide. Des questions très difficiles, avant tout pour les États et leurs citoyens, comme celles du fondement et des limites de l'autonomie d'une OI, voire de sa "souveraineté», se posent alors. La situation actuelle, c'est-à-dire l'autonomie d'OI dont les fonctions sont séparées de la souveraineté des États et de ses limites, mais sans être investies de souveraineté et de limites propres à cette souveraineté, alors même que tout leur régime de personnalité, d'immunités et de responsabilité est calqué sur celui des États, est profondément paradoxale. Ce statu quo sert les États. Leur souveraineté ne fonctionne plus comme une limite des OI, ce qui les rend irresponsables à l'égard des actes des OI, mais ces dernières, dont la personnalité, la responsabilité et les immunités sont pourtant modelées sur celles des États, ne se voient reconnaître aucune souveraineté par ceux-là, ne peuvent pas endosser les obligations ou les responsabilités qui vont de pair avec cette dernière, et ne les menacent donc pas.

Deuxièmement, sur cette base, il conviendrait d'envisager, tout en en évaluant les conséquences pour la souveraineté nationale, de nouvelles obligations primaires, puis de nouvelles formes de responsabilité internationale des OI qui leur soient davantage adaptées et soient donc plus justes.

Il faudrait, tout d'abord, repenser la personnalité internationale des OI de manière à ne pas nécessairement émuler celle des États, tout en mesurant les implications de cette émancipation pour la démocratie et la 
souveraineté étatiques. Ensuite, il conviendrait de développer des droits et obligations primaires des OI aptes à leur donner des compétences et des limites qui dépassent le champ de leurs fonctions et les angles morts de la théorie fonctionnaliste, mais sans simplement les soumettre aux mêmes droits et obligations que les États. Finalement, il faudrait développer un régime de responsabilité internationale de droit public qui soit adapté aux conséquences de la violation des nouvelles obligations primaires constitutives de la nouvelle personnalité des OI. Un modèle de responsabilité de droit public relevant de l'exercice vertical de l'autorité publique serait mieux adapté à la responsabilité des États et des OI que le modèle actuel, inspiré avant tout de la responsabilité civile ou horizontale des particuliers.

C'est à ce prix seulement que la responsabilité des États et/ou des OI pourra se développer de manière à refléter, mais aussi - et c'est une dimension sous-estimée - de manière à susciter, comme savent le faire les bonnes règles de droit, la solidarité entre ces différents acteurs de nos relations internationales. 